Homology, Homotopy and Applications, vol. 13(1), 2011, pp.89-119

\title{
ON THE 3-ARROW CALCULUS FOR HOMOTOPY CATEGORIES
}

\author{
SEBASTIAN THOMAS
}

\author{
(communicated by Charles A. Weibel)
}

\begin{abstract}
We develop a localisation theory for certain categories, yielding a 3-arrow calculus: Every morphism in the localisation is represented by a diagram of length 3 , and two such diagrams represent the same morphism if and only if they can be embedded in a 3-by-3 diagram in an appropriate way. Applications include the localisation of an arbitrary Quillen model category with respect to its weak equivalences as well as the localisation of its full subcategories of cofibrant, fibrant and bifibrant objects, giving the homotopy category in all four cases. In contrast to the approach of DWYER, HIRSCHHORN, KAN and SMith, the Quillen model category under consideration does not need to admit functorial factorisations.
\end{abstract}

\section{Introduction}

The construction of the homotopy category of a Quillen model category, that is, the localisation with respect to its set of weak equivalences, is usually done by a construction that works for arbitrary subsets of morphisms to be formally inverted, called Gabriel-Zisman localisation. However, the morphisms in the Gabriel-Zisman localisation are, in general, represented by zigzags

of finite but arbitrary length, where the "backward" arrows are in the set of those morphisms to be formally inverted. Furthermore, in the Gabriel-Zisman localisation one has, in general, no convenient criterion to decide whether two zigzags represent the same morphism in the localisation.

For a Quillen model category $\mathcal{M}$, one can do better: Recently, DwYer, HirschHORN, KAN and SMITH developed in [4, sec. 10, sec. 36] a 3-arrow calculus for the homotopy category of $\mathcal{M}$, provided $\mathcal{M}$ admits functorial factorisations (cf. $[\mathbf{4}$, sec. 9.1 , ax. MC5]). That is, they showed that each morphism in Ho $\mathcal{M}$ is represented by a

Received August 23, 2010, revised September 24, 2010; published on March 24, 2011. 2000 Mathematics Subject Classification: 18E35, 18G55, 18E30, 55U35.

Key words and phrases: localisation, 3-arrow calculus, homotopy category, derived category. Article available at http://intlpress.com/HHA/v13/n1/a4 and doi:10.4310/HHA.2011.v13.n1.a4 Copyright (C) 2011, International Press. Permission to copy for private use granted. 
diagram

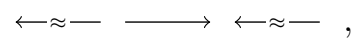

and, moreover, that two of these diagrams represent the same morphism if and only if they can be embedded as the top and the bottom row in a commutative diagram of the following form.

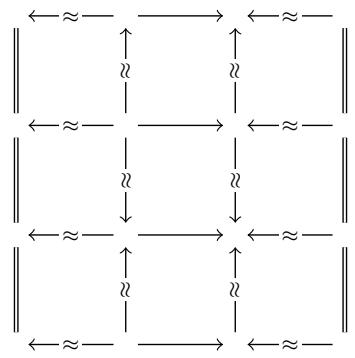

To do this, they introduced the notion of a homotopical category admitting a 3-arrow calculus $[\mathbf{4}$, sec. 33.1, 36.1] and developed a 3-arrow calculus in this context $[\mathbf{4}$, sec. 36.3].

In this article, we introduce the concept of a uni-fractionable category, see definition 3.1. Our main result is the construction of a localisation of a uni-fractionable category (with respect to its set of denominators) that satisfies a 3 -arrow calculus in the sense described above, see theorem 5.13. In contrast to [4], we will not make use of the Gabriel-Zisman localisation. Instead, we will give an elementary ad hoc construction of a localisation of a uni-fractionable category, in the spirit of the Ore localisation for a 2 -arrow calculus. $\left({ }^{1}\right)$

Both in the approach of [4, sec. 36.1] and in our uni-fractionable categories, one has three distinguished kinds of morphisms, which, in our terminology, are called denominators, S-denominators and T-denominators. The denominators are the morphisms to be formally inverted, while the S- and T-denominators are particular denominators. The essential stipulations in [4, sec. 36.1] are that every denominator factors functorially into an S-denominator followed by a T-denominator $\left({ }^{2}\right)$ and that one has functorial Ore completions along S-denominators resp. T-denominators. For uni-fractionable categories, we omit the stipulations of functoriality; instead, we require the existence of weakly universal Ore completions along S-denominators resp. T-denominators.

The advantage of uni-fractionable categories is that functoriality of factorisations is not needed. On the one hand, this is convenient for applications. On the other hand, the theory developed here can be applied to arbitrary Quillen model categories. Moreover, it can also be applied to the full subcategories of the cofibrant, fibrant resp. bifibrant objects of a Quillen model category. As a consequence, all of them admit a 3 -arrow calculus.

\footnotetext{
${ }^{1}$ It is easy to show that every morphism in the Gabriel-Zisman localisation of a uni-fractionable category can be represented by a diagram of length 3 (cf. the definition of the composition in proposition 5.2). However, the author does not know how to prove in that context that two of these diagrams represent the same morphism if and only if they can be embedded in a 3-by-3 diagram as above.

${ }^{2}$ The $\mathrm{S}$ resp. the $\mathrm{T}$ should remind us of the fact that the S-denominator resp. the T-denominator in a factorisation has the same source resp. the same target as the factorised morphism.
} 
Furthermore, a derivable category in the sense of Cisinski [2, sec. 2.25] $\left({ }^{3}\right)$, which is a self-dual generalisation of a category of fibrant objects in the sense of K. BROWN [1, sec. 1], admits a 3-arrow calculus, provided stronger variants of the factorisation axioms and the axioms which ensure stability of acyclic cofibrations under pushouts resp. of acyclic fibrations under pullbacks hold. For the relationship of Cisinski's approach with other axiom systems, see [14, sec. 2].

\section{Outline}

We recall in section 2 some notions of localisation theory and indicate how quotients of (ordered) graphs with respect to so-called graph congruences can be constructed. In section 3, uni-fractionable categories are introduced. Recall that the aim of this article is to construct a localisation of a uni-fractionable category with respect to its set of denominators. To this end, we proceed in two steps: In section 4, we assign to a uni-fractionable category a certain graph, its 3-arrow graph, and introduce a graph congruence on this graph. Then, in section 5, it turns out that the quotient graph has a canonically given category structure, and we will show that this category is a localisation of the uni-fractionable category we started with. Our main theorem 5.13 then gives a criterion on when two 3-arrows represent the same morphism in the localisation. Finally, in section 6, we show how Quillen model categories and derivable categories (under additional conditions) fit into this framework.

\section{Acknowledgements}

I thank MATTHIAS KÜNZER for many useful discussions on this article.

This article will be part of my forthcoming doctoral thesis. I thank the RWTH Aachen Graduiertenförderung for financial support.

\section{Conventions and notations}

We use the following conventions and notations.

- The composite of morphisms $f: X \rightarrow Y$ and $g: Y \rightarrow Z$ is usually denoted by $f g: X \rightarrow Z$. The composite of functors $F: \mathcal{C} \rightarrow \mathcal{D}$ and $G: \mathcal{D} \rightarrow \mathcal{E}$ is usually denoted by $G \circ F: \mathcal{C} \rightarrow \mathcal{E}$.

- Given a coproduct $C$ of $X_{1}$ and $X_{2}$, the embedding $X_{k} \rightarrow C$ is denoted by $\mathrm{emb}_{k}=\mathrm{emb}_{k}^{C}$ for $k \in\{1,2\}$. Given morphisms $f_{k}: X_{k} \rightarrow Y$ for $k \in\{1,2\}$, the induced morphism $C \rightarrow Y$ is denoted by $\left(\begin{array}{c}f_{1} \\ f_{2}\end{array}\right)=\left(\begin{array}{c}f_{1} \\ f_{2}\end{array}\right)^{C}$.

- Given an initial object $I$, the unique morphism $I \rightarrow X$ to an object $X$ will be denoted by ini $=\operatorname{ini}_{X}=\operatorname{ini}_{X}^{I}$.

- Given a category admitting finite coproducts and objects $X_{1}, X_{2}$, we denote by $X_{1} \amalg X_{2}$ a chosen coproduct and by i a chosen initial object. Analogously, given morphisms $f_{k}: X_{k} \rightarrow Y_{k}$ for $k \in\{1,2\}$, the coproduct of $f_{1}$ and $f_{2}$ is denoted by $f_{1} \amalg f_{2}$.

- Given a category admitting finite coproducts $\mathcal{C}$ and a category $\mathcal{D}$, we say that a functor $F: \mathcal{C} \rightarrow \mathcal{D}$ preserves finite coproducts if $F_{\mathrm{i}}$ is an initial object in $\mathcal{D}$, and if, given $X_{1}, X_{2} \in \mathrm{ObC}$, the object $F\left(X_{1} \amalg X_{2}\right)$ is a coproduct of $F X_{1}$ and $F X_{2}$, where the embeddings are given by $\mathrm{emb}_{k}^{F\left(X_{1} \amalg X_{2}\right)}=F\left(\mathrm{emb}_{k}^{X_{1} \amalg X_{2}}\right)$ for $k \in\{1,2\}$.

\footnotetext{
${ }^{3}$ Also called an Anderson-Brown-Cisinski premodel category by RăDULESCU-BANU [14, def. 1.1.3].
} 
- By a weak pushout rectangle (resp. weak pullback rectangle) we understand a quadrangle having the universal property of a pushout rectangle (resp. pullback rectangle) except for the uniqueness of the induced morphism.

- Given integers $a, b \in \mathbb{Z}$, we write $[a, b]:=\{z \in \mathbb{Z} \mid a \leqslant z \leqslant b\}$ for the set of integers lying between $a$ and $b$.

\section{Preliminaries}

In this section, we give some preliminaries on localisations of categories and quotient graphs with respect to graph congruences.

\section{Localisations of categories}

We suppose given a category $\mathcal{C}$. A denominator set in $\mathcal{C}$ is a subset $D \subseteq \operatorname{Mor} \mathcal{C}$. We will consider denominator sets with special properties later in this article, but at the moment, a denominator set $D$ is just an arbitrary subset of Mor $\mathcal{C}$. Informally, it is a subset singled out with the "intention of localising with respect to it", in the following sense.

A localisation of $\mathcal{C}$ with respect to a denominator set $D$ in $\mathcal{C}$ consists of a category $\mathcal{L}$ and a functor $L: \mathcal{C} \rightarrow \mathcal{L}$ such that the following axioms hold.

(Inv) Invertibility. For all $d \in D$, the morphism $L d$ is invertible.

(1-uni) 1-universality. Given a category $\mathcal{D}$ and a functor $F: \mathcal{C} \rightarrow \mathcal{D}$ such that $F d$ is invertible for all $d \in D$, there exists a unique functor $\hat{F}: \mathcal{L} \rightarrow \mathcal{D}$ with $F=\hat{F} \circ L$.

(2-uni) 2-universality. We suppose given a category $\mathcal{D}$ and functors $F, G: \mathcal{C} \rightarrow \mathcal{D}$ such that $F d$ and $G d$ are invertible for all $d \in D$, and we denote by $\hat{F}: \mathcal{L} \rightarrow \mathcal{D}$ resp. $\hat{G}: \mathcal{L} \rightarrow \mathcal{D}$ the unique functor with $F=\hat{F} \circ L$ resp. $G=\hat{G} \circ L$. Given a transformation $\alpha: F \rightarrow G$, there exists a unique transformation $\hat{\alpha}: \hat{F} \rightarrow \hat{G}$ such that $\hat{\alpha}_{L X}=\alpha_{X}$ for all $X \in \mathrm{Ob} \mathcal{C}$.

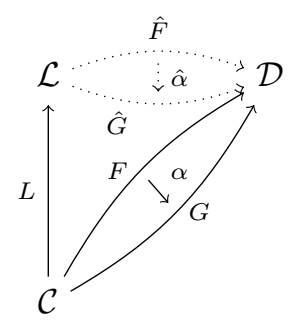

By abuse of notation, we refer to the localisation as well as to its underlying category just by $\mathcal{L}$. The functor $L$ is said to be the localisation functor of the localisation $\mathcal{L}$. Given a localisation $\mathcal{L}$ of $\mathcal{C}$ with respect to $D$ with localisation functor $L: \mathcal{C} \rightarrow \mathcal{L}$, we write $\operatorname{loc}=\operatorname{loc}^{\mathcal{L}}:=L$.

GABRIEL and ZiSMAN have shown in [7, sec. 1.1] that there exists a localisation of every category $\mathcal{C}$ with respect to an arbitrary denominator set $D$ in $\mathcal{C}$. We will not make use of this result. Rather, given a uni-fractionable category, see definition 3.1, we construct a localisation directly, cf. propositions 5.2 and 5.5. 


\section{Saturatedness}

We suppose given a category $\mathcal{C}$, a denominator set $D$ in $\mathcal{C}$, and a localisation $\mathcal{L}$ of $\mathcal{C}$ with respect to $D$. By definition of a localisation, $\operatorname{loc}(d)$ is invertible for every $d \in D$. But in general, not every morphism $f$ in $\mathcal{C}$ for which $\operatorname{loc}(f)$ is invertible in $\mathcal{L}$ has to be an element of $D$. The denominator set $D$ is said to be saturated if $f \in D$ for all $f \in \operatorname{Mor} \mathcal{C}$ with $\operatorname{loc}(f)$ invertible in $\mathcal{L}$. We use the following notions to indicate how far $D$ is away from this property.

The denominator set $D$ is said to be multiplicative if it fulfills:

(Cat) Multiplicativity. For all $d, e \in D$ with Target $d=$ Source $e$, their composite de is in $D$, and for every object $X$ in $\mathcal{C}$, the identity $1_{X}$ is in $D$.

The denominator set $D$ is said to be semi-saturated if it is multiplicative and fulfills:

(2 of 3 ) 2 out of 3 axiom. We suppose given morphisms $f$ and $g$ in $\mathcal{C}$ with Target $f=$ Source $g$. If two out of the morphisms $f, g, f g$ are in $D$, then so is the third.

Finally, the denominator set $D$ is said to be weakly saturated if it is multiplicative and fulfills:

(2 of 6) 2 out of 6 axiom. We suppose given morphisms $f, g, h$ in $\mathcal{C}$ with Target $f=$ Source $g$ and Target $g=$ Source $h$. If $f g, g h \in D$, then $f, g, h, f g h \in D$.

Saturatedness implies weak saturatedness, weak saturatedness implies semi-saturatedness, and semi-saturatedness implies multiplicativity (the last impliciation holds by definition).

\section{Graph congruences and quotient graphs}

We suppose given an (oriented) graph $\mathcal{G}$. An equivalence relation $\equiv$ on $\operatorname{Arr} \mathcal{G}$ is said to be a graph congruence on $\mathcal{G}$ if Source $a=$ Source $\tilde{a}$ and Target $a=$ Target $\tilde{a}$ for all $a, \tilde{a} \in \operatorname{Arr} \mathcal{G}$ with $a \equiv \tilde{a}$. Given a graph congruence $\equiv$ on $\mathcal{G}$, the quotient graph of $\mathcal{G}$ with respect to $\equiv$ is the graph $\mathcal{G} / \equiv$ with $\operatorname{Ob} \mathcal{G} / \equiv:=\operatorname{Ob} \mathcal{G}, \operatorname{Arr} \mathcal{G} / \equiv:=(\operatorname{Arr} \mathcal{G}) / \equiv$ and Source $[a]_{\equiv}:=$ Source $a$, Target $[a]_{\equiv}:=$ Target $a$ for $a \in \operatorname{Arr} \mathcal{G}$. The graph morphism quo $=\operatorname{quo}^{\mathcal{G}} / \equiv: \mathcal{G} \rightarrow \mathcal{G} / \equiv$ given by $q u o(X):=X$ and $q u o(a):=[a]_{\equiv}$ is called the quotient graph morphism.

The quotient graph of $\mathcal{G}$ with respect to a graph congrunce $\equiv$ fulfills the following universal property. Given $a, \tilde{a} \in \operatorname{Arr} \mathcal{G}$ with $a \equiv \tilde{a}$, we have quo $(a)=$ quo $(\tilde{a})$. For every graph $\mathcal{H}$ and every graph morphism $F: \mathcal{G} \rightarrow \mathcal{H}$ with $F a=F \tilde{a}$ for $a, \tilde{a} \in \operatorname{Arr} \mathcal{G}$ with $a \equiv \tilde{a}$, there exists a unique graph morphism $\bar{F}: \mathcal{G} / \equiv \rightarrow \mathcal{H}$ with $F=\bar{F}$ ○ quo.

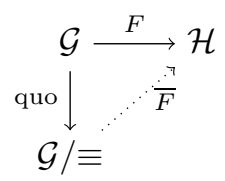

\section{Uni-fractionable categories}

Definition 3.1 (uni-fractionable category). A uni-fractionable category $\left({ }^{4}\right)$ consists of a category $\mathcal{C}$ together with a semi-saturated denominator set $D$ in $\mathcal{C}$ and multiplicative subsets $S, T \subseteq D$, such that the following axioms hold.

\footnotetext{
${ }^{4}$ There exists also the notion of a fractionable category, cf. the author's forthcoming doctoral thesis.
} 
(WU) Weakly universal Ore completions. Given morphisms $i$ and $f$ in $\mathcal{C}$ with $i \in S$ and Source $i=$ Source $f$, there exists a weak pushout rectangle in $\mathcal{C}$ as displayed below on the left, such that $i^{\prime} \in S$. Dually, given morphisms $p$ and $f$ in $\mathcal{C}$ with $p \in T$ and Target $p=$ Target $f$, there exists a weak pullback rectangle in $\mathcal{C}$ as displayed below on the right, such that $p^{\prime} \in T$.
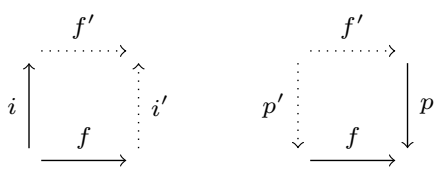

(Fac) Factorisations. For every $d \in D$, there exist $i \in S$ and $p \in T$ with $d=i p$.

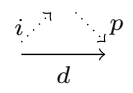

By abuse of notation, we refer to the uni-fractionable category as well as to its underlying category just by $\mathcal{C}$. The elements of $D$ resp. $S$ resp. $T$ are called denominators resp. S-denominators resp. T-denominators in $\mathcal{C}$.

Given a uni-fractionable category $\mathcal{C}$ with set of denominators $D$, set of S-denominators $S$ and set of T-denominators $T$, we write $\operatorname{Den} \mathcal{C}:=D, \operatorname{SDen} \mathcal{C}:=S$, TDen $\mathcal{C}:=T$. In diagrams, a denominator $d$ resp. an S-denominator $i$ resp. a T-denominator $p$ in $\mathcal{C}$ will usually be depicted as

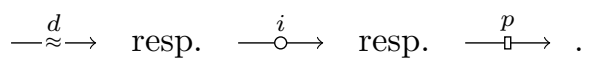

Some examples of uni-fractionable categories can be found in section 6 .

Definition 3.2 (denominator preserving functor). Given uni-fractionable categories $\mathcal{C}$ and $\mathcal{D}$, a functor $F: \mathcal{C} \rightarrow \mathcal{D}$ is said to preserve denominators if $F d$ is a denominator in $\mathcal{D}$ for every denominator $d$ in $\mathcal{C}$.

\section{The 3-arrow graph}

We want to construct a localisation $\operatorname{Frac} \mathcal{C}$ of a uni-fractionable category $\mathcal{C}$ with respect to its set of denominators Den $\mathcal{C}$. To this end, we begin in this section by introducing its 3 -arrow graph $\mathrm{AGC}$ and a graph congruence $\equiv$ on $\mathrm{AGC}$.

In this section, we suppose given a uni-fractionable category $\mathcal{C}$.

Definition 4.1 (3-arrow shape). The graph

$$
0 \stackrel{\tau}{\longleftarrow} 1 \stackrel{v}{\longrightarrow} 2 \stackrel{\sigma}{\longleftarrow} 3
$$

is said to be the 3-arrow shape and will be denoted by $\boldsymbol{\Theta}$.

Recall that a diagram of shape $\Theta$ in $\mathcal{C}$ is just a graph morphism $A: \Theta \rightarrow \mathcal{C}$. Given a diagram $D$ of shape $\boldsymbol{\Theta}$ in $\mathcal{C}$, we write $D_{i}:=D(i)$ for $i \in \mathrm{Ob} \Theta$ and $D_{a}:=D(a)$ for $a \in \operatorname{Arr} \Theta$. Given diagrams $D$ and $E$, a diagram morphism from $D$ to $E$ is a family $f=\left(f_{i}\right)_{i \in \mathrm{Ob} \Theta}$ in $\operatorname{Mor} \mathcal{C}$ with $D_{a} f_{j}=f_{i} E_{a}$ for all arrows $a: i \rightarrow j$ in $\Theta$. The category consisting of diagrams of shape $\boldsymbol{\Theta}$ in $\mathcal{C}$ as objects and diagram morphisms between those diagrams as morphisms will be denoted by $\mathcal{C}^{\Theta}$. 
Definition 4.2 (3-arrow graph). The 3-arrow graph of $\mathcal{C}$ is defined to be the graph $\mathrm{AGC}$ with object set $\mathrm{Ob} \mathrm{AGC}:=\mathrm{Ob} \mathcal{C}$ and arrow set Arr AGC $:=\left\{A \in \mathrm{Ob} \mathcal{C}^{\Theta} \mid A_{\sigma}\right.$, $\left.A_{\tau} \in \operatorname{Den} \mathcal{C}\right\}$. The source resp. the target of $A \in \operatorname{Arr}$ AG $\mathcal{C}$ are defined by Source $A:=$ $A_{0}$ resp. Target $A:=A_{3}$.

An arrow $A$ in $\mathrm{AGC}$ is called a 3 -arrow in $\mathcal{C}$. Given a denominator $b: \tilde{X} \rightarrow X$, a morphism $f: \tilde{X} \rightarrow \tilde{Y}$ and a denominator $a: Y \rightarrow \tilde{Y}$ in $\mathcal{C}$, we abuse notation and denote the unique 3 -arrow $A$ with $A_{\tau}=b, A_{v}=f, A_{\sigma}=a$ by $(b, f, a):=A$. Moreover, we use the notation $(b, f, a): X \leftarrow \tilde{X} \rightarrow \tilde{Y} \leftarrow Y$.

$$
X \longleftarrow \stackrel{b}{\approx}-\tilde{X} \stackrel{f}{\longrightarrow} \tilde{Y} \stackrel{a}{\longleftarrow}-Y
$$

Our next step will be the introduction of an equivalence relation on the arrow set of the 3-arrow graph.

Definition 4.3 (fraction equality). The equivalence relation $\equiv$ on $\mathrm{Arr} A G \mathcal{C}$ is defined to be generated by the following relation on $\operatorname{Arr} A G \mathcal{C}$ : Given $(b, f, a) \in \operatorname{Arr} A G \mathcal{C}$ and $c \in \operatorname{Mor} \mathcal{C}$ with $a c \in \operatorname{Den} \mathcal{C}$, the 3 -arrow $(b, f, a)$ is in relation to the 3 -arrow $(b, f c, a c)$; and given $(b, f, a) \in \operatorname{Arr} \operatorname{AGC}$ and $c \in \operatorname{Mor} \mathcal{C}$ with $c b \in \operatorname{Den} \mathcal{C}$, the 3 -arrow $(b, f, a)$ is in relation to the 3 -arrow $(c b, c f, a)$.
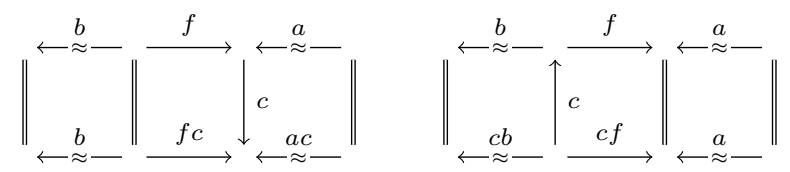

Given $(b, f, a),(\tilde{b}, \tilde{f}, \tilde{a}) \in \operatorname{Arr} \operatorname{AGC}$ with $(b, f, a) \equiv(\tilde{b}, \tilde{f}, \tilde{a})$, we say that $(b, f, a)$ and $(\tilde{b}, \tilde{f}, \tilde{a})$ are fraction equal.

In practice, it is sometimes convenient to work with different generating sets for fraction equality.

Remark 4.4. (a) The fraction equality relation $\equiv$ on Arr AGC $\mathcal{C}$ is generated by the following relation: Given $(b, f, a) \in \operatorname{Arr} \mathrm{AGC}$ and $c, c^{\prime} \in \operatorname{Mor} \mathcal{C}$ with $a c, c^{\prime} b \in \operatorname{Den} \mathcal{C}$, the 3 -arrow $(b, f, a)$ is in relation to the 3 -arrow $\left(c^{\prime} b, c^{\prime} f c, a c\right)$.

(b) The fraction equality relation $\equiv$ on Arr AGC is generated by the following relation: Given $(b, f, a),(\tilde{b}, \tilde{f}, \tilde{a}) \in \operatorname{Arr} \mathrm{AGC}$, the 3 -arrow $(b, f, a)$ is in relation to the 3-arrow $(\tilde{b}, \tilde{f}, \tilde{a})$ if there exist $c, c^{\prime} \in \operatorname{Mor} \mathcal{C}$ with $b=c^{\prime} \tilde{b}, f c=c^{\prime} \tilde{f}, a c=\tilde{a}$.
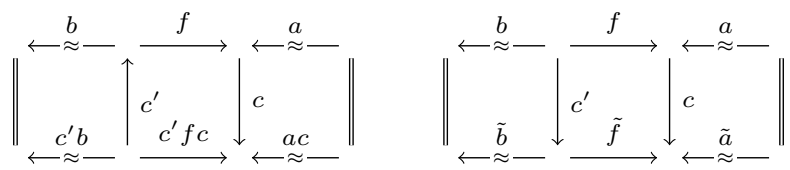

As Den $\mathcal{C}$ is semi-saturated, the morphisms $c$ and $c^{\prime}$ in definition 4.3 and remark 4.4 are automatically denominators in $\mathcal{C}$.

Remark 4.5. We suppose given 3 -arrows $(b, f, a)$ and $(\tilde{b}, \tilde{f}, \tilde{a})$ in $\mathcal{C}$. If $(b, f, a) \equiv$ $(\tilde{b}, \tilde{f}, \tilde{a})$, then $f$ is a denominator in $\mathcal{C}$ if and only if $\tilde{f}$ is a denominator in $\mathcal{C}$.

Proof. This follows by the definition of fraction equality 4.3 and by the semi-saturatedness of Den $\mathcal{C}$. 
Remark 4.6. The fraction equality relation $\equiv$ on Arr AGC defines a graph congruence on $\mathrm{AGC}$. In particular, the quotient graph $(\mathrm{AGC}) / \equiv$ is defined.

Proof. For $(b, f, a) \in \operatorname{Arr} \operatorname{AGC}, c, c^{\prime} \in \operatorname{Mor} \mathcal{C}$ with $a c, c^{\prime} b \in \operatorname{Den} \mathcal{C}$, we have

$$
\text { Source }\left(c^{\prime} b, c^{\prime} f c, a c\right)=\operatorname{Target}\left(c^{\prime} b\right)=\operatorname{Target} b=\operatorname{Source}(b, f, a)
$$

and analogously Target $\left(c^{\prime} b, c^{\prime} f c, a c\right)=$ Target $(b, f, a)$. Thus the assertion follows from remark 4.4(a).

Definition 4.7 (double fraction). Given a 3 -arrow $(b, f, a)$ in $\mathcal{C}$, its equivalence class in the quotient graph $(\mathrm{AGC}) / \equiv$ is denoted by $b \backslash f / a:=[(b, f, a)]_{\equiv}$ and is said to be the double fraction of $(b, f, a)$.

Now we will present a certain reduced form for 3-arrows. We will see that every 3 -arrow is fraction equal to such a reduced form.

Definition 4.8 (normal 3-arrows). A 3 -arrow $(p, f, i)$ in $\mathcal{C}$ is said to be normal if $i$ is an $\mathrm{S}$-denominator and $p$ is a $\mathrm{T}$-denominator in $\mathcal{C}$.

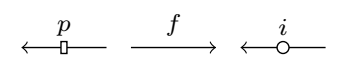

The following lemma and its proof is (essentially) taken from [4, sec. 36.5].

Lemma 4.9 (normalisation lemma). Every 3 -arrow in $\mathcal{C}$ is fraction equal to a normal 3-arrow in $\mathcal{C}$.

Proof. We suppose given an arbitrary 3 -arrow $(b, f, a)$ in $\mathcal{C}$. There exist an S-denominator $i$ and a T-denominator $p$ in $\mathcal{C}$ with $b=i p$, and there exist an S-denominator $i^{\prime}$ and a morphism $f^{\prime}$ in $\mathcal{C}$ with $i f^{\prime}=f i^{\prime}$. By multiplicativity, $a i^{\prime}$ is a denominator in $\mathcal{C}$. Thus there exist an S-denominator $j$ and a T-denominator $q$ in $\mathcal{C}$ with $a i^{\prime}=j q$, and there exist a T-denominator $q^{\prime}$ and a morphism $f^{\prime \prime}$ in $\mathcal{C}$ with $f^{\prime \prime} q=q^{\prime} f^{\prime}$. By multiplicativity, $q^{\prime} p$ is a T-denominator.

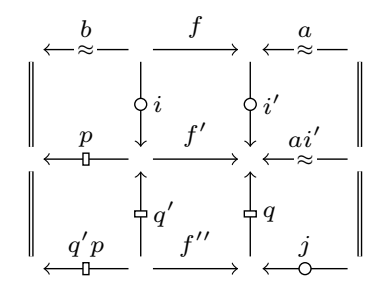

Altogether, $(b, f, a) \equiv\left(p, f^{\prime}, a i^{\prime}\right) \equiv\left(q^{\prime} p, f^{\prime \prime}, j\right)$, and since $j$ is an S-denominator and $q^{\prime} p$ is a T-denominator, the 3 -arrow $\left(q^{\prime} p, f^{\prime \prime}, j\right)$ is normal.

\section{The fraction category}

In this section, our main theorem 5.13 will be proven. We begin by constructing a localisation of a uni-fractionable category $\mathcal{C}$ with respect to its set of denominators Den $\mathcal{C}$, see proposition 5.2 and proposition 5.5. To this end, we consider the quotient graph $(\mathrm{AGC}) / \equiv$ of its 3 -arrow graph $\mathrm{AGC}$ with respect to fraction equality $\equiv$. The crucial point in the construction will be the following lemma.

Troughout this section, we suppose given a uni-fractionable category $\mathcal{C}$. 
Lemma 5.1 (factorisation lemma). We suppose given denominators $d$, e and morphisms $f, g$ in $\mathcal{C}$ with $f e=d g$. Moreover, we suppose given $S$-denominators $i, j$ and $T$-denominators $p, q$ in $\mathcal{C}$ with $d=i p$ and $e=j q$. Then there exist $S$-denominators $\tilde{j}, k, a$ T-denominator $\tilde{q}$ and a morphism $h$ in $\mathcal{C}$ such that $e=\tilde{j} \tilde{q}, f \tilde{j}=i h, p g=h \tilde{q}$, $\tilde{j}=j k, q=k \tilde{q}$.

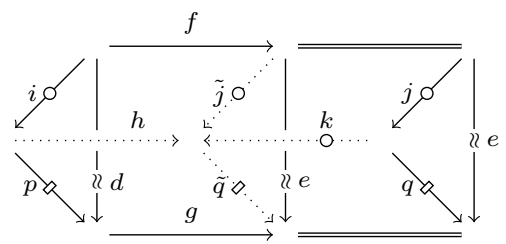

Proof. We let

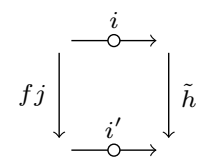

be a weak pushout rectangle in $\mathcal{C}$ such that $i^{\prime}$ is an S-denominator in $\mathcal{C}$. Since $i p g=$ $d g=f e=f j q$, there exists an induced morphism $a$ with $q=i^{\prime} a$ and $p g=\tilde{h} a$. By semi-saturatedness, $a$ is a denominator in $\mathcal{C}$, and thus there exist an S-denominator $\tilde{k}$ and a T-denominator $\tilde{q}$ with $a=\tilde{k} \tilde{q}$.

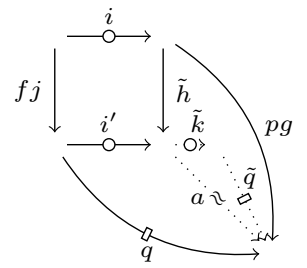

We set $h:=\tilde{h} \tilde{k}, k:=i^{\prime} \tilde{k}, \tilde{j}:=j i^{\prime} \tilde{k}$ and get $e=\tilde{j} \tilde{q}, f \tilde{j}=i h, p g=h \tilde{q}, \tilde{j}=j k, q=k \tilde{q}$. Moreover, $k=i^{\prime} \tilde{k}$ and $\tilde{j}=j i^{\prime} \tilde{k}$ are S-denominators in $\mathcal{C}$ by multiplicativity.

The following proposition will essentially prove the first part of our main theorem 5.13, cf. also proposition 5.7 below.

Proposition 5.2. There is a category structure on $(\mathrm{AGC}) / \equiv$, where the composition is constructed by the following procedure.

We suppose given $\left(b_{1}, f_{1}, a_{1}\right),\left(b_{2}, f_{2}, a_{2}\right) \in \operatorname{Arr}$ AGC with Target $\left(b_{1}, f_{1}, a_{1}\right)=$ Source $\left(b_{2}, f_{2}, a_{2}\right)$. First, we choose an S-denominator $j$ and a T-denominator $q$ in $\mathcal{C}$ with $b_{2} a_{1}=j q$. Second, we choose a T-denominator $q^{\prime}$ and a morphism $f_{1}^{\prime}$ in $\mathcal{C}$ with $f_{1}^{\prime} q=q^{\prime} f_{1}$, and we choose an $S$-denominator $j^{\prime}$ and a morphism $f_{2}^{\prime}$ in $\mathcal{C}$ with $j f_{2}^{\prime}=f_{2} j^{\prime}$. Then $\left(b_{1} \backslash f_{1} / a_{1}\right)\left(b_{2} \backslash f_{2} / a_{2}\right)=q^{\prime} b_{1} \backslash f_{1}^{\prime} f_{2}^{\prime} / a_{2} j^{\prime}$.

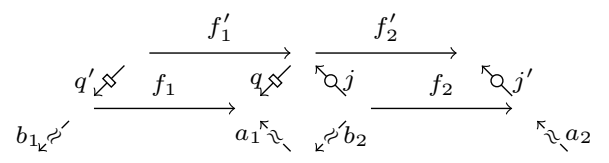

The identity of $X \in \mathrm{Ob}(\mathrm{AGC}) / \equiv$ is given by $1_{X}=1_{X} \backslash 1_{X} / 1_{X}$. 
Proof. Our first aim is to show that the construction described above is independent of all choices. To this end, we first consider the particular case of choosing a weak pullback of $f_{1}$ and $q$ and a weak pushout of $f_{2}$ and $j$ to obtain a T-denominator $q^{\prime}$, an S-denominator $j^{\prime}$ and morphisms $f_{1}^{\prime}, f_{2}^{\prime}$ in $\mathcal{C}$.

We suppose given $\left(b_{l}, f_{l}, a_{l}\right),\left(\tilde{b}_{l}, \tilde{f}_{l}, \tilde{a}_{l}\right) \in \operatorname{Arr} \operatorname{AGC}, c_{l}, c_{l}^{\prime} \in \operatorname{Mor} \mathcal{C}$ with $b_{l}=c_{l} \tilde{b}_{l}, f_{l} c_{l}^{\prime}$ $=c_{l} \tilde{f}_{l}, a_{l} c_{l}^{\prime}=\tilde{a}_{l}$ for $l \in\{1,2\}$, and such that Target $\left(b_{1}, f_{1}, a_{1}\right)=\operatorname{Source}\left(b_{2}, f_{2}, a_{2}\right)$.

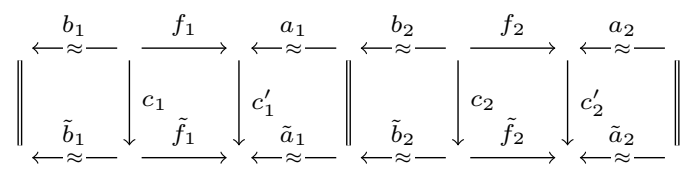

We choose S-denominators $j, \tilde{j}$ and T-denominators $q, \tilde{q}$ in $\mathcal{C}$ such that $b_{2} a_{1}=j q$ and $\tilde{b}_{2} \tilde{a}_{1}=\tilde{j} \tilde{q}$. By the factorisation lemma 5.1, there exist an S-denominator $k$, a T-denominator $r$ and morphisms $c, \tilde{c}$ in $\mathcal{C}$ with $\tilde{b}_{2} \tilde{a}_{1}=k r, q c_{1}^{\prime}=c r, c_{2} k=j c, \tilde{q}=\tilde{c} r$, $k=\tilde{j} \tilde{c}$. Next, we choose weak pullback rectangles

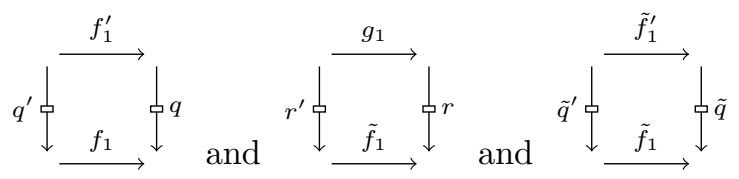

in $\mathcal{C}$ such that $q^{\prime}, r^{\prime}, \tilde{q}^{\prime}$ are T-denominators, and we choose weak pushout rectangles

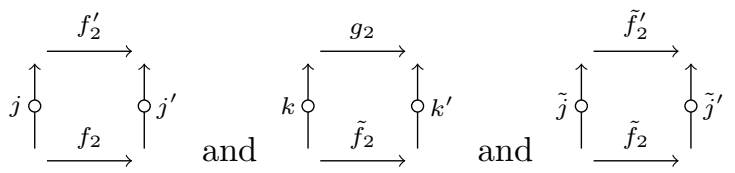

in $\mathcal{C}$ such that $j^{\prime}, k^{\prime}, \tilde{j}^{\prime}$ are S-denominators. We obtain induced morphisms $c^{\prime}$ and $\tilde{c}^{\prime}$ on the weak pullbacks, that is, with $q^{\prime} c_{1}=c^{\prime} r^{\prime}, f_{1}^{\prime} c=c^{\prime} g_{1}$ and $\tilde{q}^{\prime}=c^{\prime} r^{\prime}, \tilde{f}_{1}^{\prime} \tilde{c}=\tilde{c}^{\prime} g_{1}$, and induced morphisms $c^{\prime \prime}$ and $\tilde{c}^{\prime \prime}$ on the weak pushouts, that is, with $c_{2}^{\prime} k^{\prime}=j^{\prime} c^{\prime \prime}$, $c g_{2}=f_{2}^{\prime} c^{\prime \prime}$ and $k^{\prime}=\tilde{j}^{\prime} \tilde{c}^{\prime \prime}, \tilde{c} g_{2}=\tilde{f}_{2}^{\prime} \tilde{c}^{\prime \prime}$.

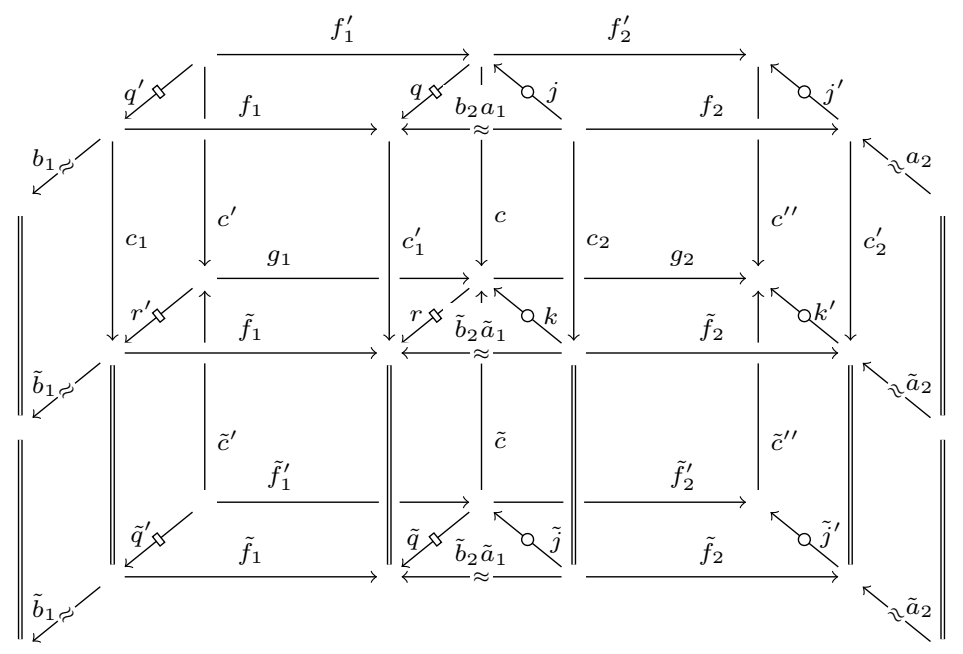


We get $q^{\prime} b_{1}=c^{\prime} r^{\prime} \tilde{b}_{1}, f_{1}^{\prime} f_{2}^{\prime} c^{\prime \prime}=c^{\prime} g_{1} g_{2}, a_{2} j^{\prime} c^{\prime \prime}=\tilde{a}_{2} k^{\prime}, \tilde{q}^{\prime} \tilde{b}_{1}=\tilde{c}^{\prime} r^{\prime} \tilde{b}_{1}, \tilde{f}_{1}^{\prime} \tilde{f}_{2}^{\prime} \tilde{c}^{\prime \prime}=\tilde{c}^{\prime} g_{1} g_{2}$, $\tilde{a}_{2} \tilde{j}^{\prime} \tilde{c}^{\prime \prime}=\tilde{a}_{2} k^{\prime}$ and therefore $\left(q^{\prime} b_{1}, f_{1}^{\prime} f_{2}^{\prime}, a_{2} j^{\prime}\right) \equiv\left(r^{\prime} \tilde{b}_{1}, g_{1} g_{2}, \tilde{a}_{2} k^{\prime}\right) \equiv\left(\tilde{q}^{\prime} \tilde{b}_{1}, \tilde{f}_{1}^{\prime} \tilde{f}_{2}^{\prime}, \tilde{a}_{2} \tilde{j}^{\prime}\right)$.

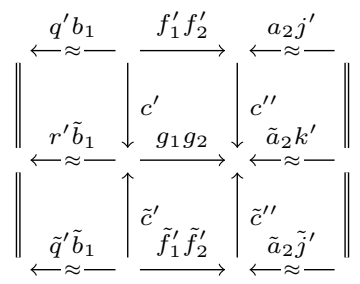

Thus we have $q^{\prime} b_{1} \backslash f_{1}^{\prime} f_{2}^{\prime} / a_{2} j^{\prime}=\tilde{q}^{\prime} \tilde{b}_{1} \backslash \tilde{f}_{1}^{\prime} \tilde{f}_{2}^{\prime} / \tilde{a}_{2} \tilde{j}^{\prime}$ in $(\mathrm{AGC}) / \equiv$.

In the special case where $c_{1}=1, c_{1}^{\prime}=1, c_{2}=1, c_{2}^{\prime}=1$, we see that different choices of constructions via weak pullback and weak pushout rectangles lead to the same double fraction $q^{\prime} b_{1} \backslash f_{1}^{\prime} f_{2}^{\prime} / a_{2} j^{\prime}=\tilde{q}^{\prime} b_{1} \backslash \tilde{f}_{1}^{\prime} \tilde{f}_{2}^{\prime} / \tilde{a}_{2} \tilde{j}^{\prime}$. Hence we obtain a well-defined map

$$
\begin{gathered}
c: \operatorname{Arr} \operatorname{AGC}_{\text {Target }} \times_{\text {Source }} \operatorname{Arr~AG\mathcal {C}} \rightarrow \operatorname{Arr}(\operatorname{AGC}) / \equiv, \\
\left(b_{1}, f_{1}, a_{1}\right),\left(b_{2}, f_{2}, a_{2}\right) \mapsto q^{\prime} b_{1} \backslash f_{1}^{\prime} f_{2}^{\prime} / a_{2} j^{\prime},
\end{gathered}
$$

where $q^{\prime}, f_{1}^{\prime}, f_{2}^{\prime}, j^{\prime}$ are constructed as described above. Now the general case shows that $c$ is independent of the choice of the representatives in the equivalence classes with respect to $\equiv$, and thus we obtain an induced map

$$
\bar{c}: \operatorname{Arr}(\mathrm{AGC}) / \equiv_{\text {Target }} \times_{\text {Source }} \operatorname{Arr}(\operatorname{AGC}) / \equiv \rightarrow \operatorname{Arr}(\operatorname{AGC}) / \equiv
$$

given by $\bar{c}\left(b_{1} \backslash f_{1} / a_{1}, b_{2} \backslash f_{2} / a_{2}\right)=c\left(\left(b_{1}, f_{1}, a_{1}\right),\left(b_{2}, f_{2}, a_{2}\right)\right)=q^{\prime} b_{1} \backslash f_{1}^{\prime} f_{2}^{\prime} / a_{2} j^{\prime}$.

We claim that arbitrary commutative quadrangles may be used instead of weak pullback and weak pushout rectangles to compute $\bar{c}$. Indeed, given a weak pullback rectangle and a weak pushout rectangle

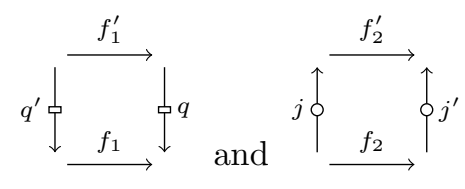

and arbitrary commutative quadrangles

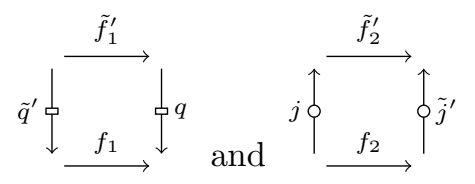

such that $q^{\prime}, \tilde{q}^{\prime}$ are T-denominators and $j^{\prime}, \tilde{j}^{\prime}$ are S-denominators in $\mathcal{C}$, we obtain induced morphisms $c$ and $c^{\prime}$ such that $\tilde{q}^{\prime}=c q^{\prime}, \tilde{f}_{1}^{\prime}=c f_{1}^{\prime}, \tilde{f}_{2}^{\prime}=f_{2}^{\prime} c^{\prime}, \tilde{j}^{\prime}=j^{\prime} c^{\prime}$.

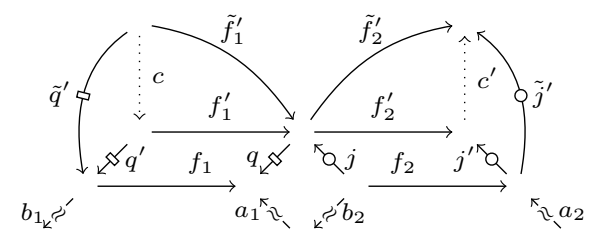


Thus we have $\left(\tilde{q}^{\prime} b_{1}, \tilde{f}_{1}^{\prime} \tilde{f}_{2}^{\prime}, a_{2} \tilde{j}^{\prime}\right)=\left(c q^{\prime} b_{1}, c f_{1}^{\prime} f_{2}^{\prime} c^{\prime}, a_{2} j^{\prime} c^{\prime}\right) \equiv\left(q^{\prime} b_{1}, f_{1}^{\prime} f_{2}^{\prime}, a_{2} j^{\prime}\right)$ and therefore $\bar{c}\left(b_{1} \backslash f_{1} / a_{1}, b_{2} \backslash f_{2} / a_{2}\right)=q^{\prime} b_{1} \backslash f_{1}^{\prime} f_{2}^{\prime} / a_{2} j^{\prime}=\tilde{q}^{\prime} b_{1} \backslash \tilde{f}_{1}^{\prime} \tilde{f}_{2}^{\prime} / a_{2} \tilde{j}^{\prime}$. This proves the claim.

In addition to $\bar{c}$, we define the map

$$
e: \operatorname{Ob}(\mathrm{AGC}) / \equiv \rightarrow \operatorname{Arr}(\mathrm{AGC}) / \equiv, X \mapsto 1_{X} \backslash 1_{X} / 1_{X} .
$$

To show that $(\mathrm{AGC}) / \equiv$ is a category with composition $\bar{c}$ and identity map $e$, it remains to verify the category axioms. By the definitions of $\bar{c}$ and $e$, we have

$$
\text { Source } \begin{aligned}
\bar{c}\left(b_{1} \backslash f_{1} / a_{1}, b_{2} \backslash f_{2} / a_{2}\right) & =\text { Source } q^{\prime} b_{1} \backslash f_{1}^{\prime} f_{2}^{\prime} / a_{2} j^{\prime}=\operatorname{Target}\left(q^{\prime} b_{1}\right)=\text { Target } b_{1} \\
& =\text { Source } b_{1} \backslash f_{1} / a_{1}
\end{aligned}
$$

and analogously Target $\bar{c}\left(b_{1} \backslash f_{1} / a_{1}, b_{2} \backslash f_{2} / a_{2}\right)=$ Target $b_{2} \backslash f_{2} / a_{2}$ for all $\left(b_{1}, f_{1}, a_{1}\right)$, $\left(b_{2}, f_{2}, a_{2}\right) \in \operatorname{Arr}$ AGC $\mathcal{C}$ with Target $b_{1} \backslash f_{1} / a_{1}=$ Source $b_{2} \backslash f_{2} / a_{2}$, as well as

$$
\text { Source } e(X)=\text { Source } 1_{X} \backslash 1_{X} / 1_{X}=\text { Target } 1_{X}=X
$$

and analogously Target $e(X)=X$ for all $X \in \mathrm{Ob}(\mathrm{AGC}) / \equiv$.

For the associativity of $\bar{c}$, we suppose given $\left(b_{l}, f_{l}, a_{l}\right) \in \operatorname{Arr}$ AGC for $l \in\{1,2,3\}$ such that Target $b_{1} \backslash f_{1} / a_{1}=$ Source $b_{2} \backslash f_{2} / a_{2}$ and Target $b_{2} \backslash f_{2} / a_{2}=$ Source $b_{3} \backslash f_{3} / a_{3}$. We choose S-denominators $j, \tilde{j}$ and T-denominators $q, \tilde{q}$ with $b_{2} a_{1}=j q$ and $b_{3} a_{2}=\tilde{j} \tilde{q}$. Then we choose T-denominators $q^{\prime}, \tilde{q}^{\prime}$ and morphisms $f_{1}^{\prime}, \tilde{f}_{2}^{\prime}$ in $\mathcal{C}$ with $f_{1}^{\prime} q=q^{\prime} f_{1}$ and $\tilde{f}_{2}^{\prime} \tilde{q}=\tilde{q}^{\prime} f_{2}$, and we choose S-denominators $j^{\prime}, \tilde{j}^{\prime}$ and morphisms $f_{2}^{\prime}, \tilde{f}_{3}^{\prime}$ in $\mathcal{C}$ with $j f_{2}^{\prime}=f_{2} j^{\prime}$ and $\tilde{j} \tilde{f}_{3}^{\prime}=f_{3} \tilde{j}^{\prime}$. By definition of $\bar{c}$, we obtain $\bar{c}\left(b_{1} \backslash f_{1} / a_{1}, b_{2} \backslash f_{2} / a_{2}\right)=$ $q^{\prime} b_{1} \backslash f_{1}^{\prime} f_{2}^{\prime} / a_{2} j^{\prime}$ and $\bar{c}\left(b_{2} \backslash f_{2} / a_{2}, b_{3} \backslash f_{3} / a_{3}\right)=\tilde{q}^{\prime} b_{2} \backslash \tilde{f}_{2}^{\prime} \tilde{f}_{3}^{\prime} / a_{3} \tilde{j}^{\prime}$.

Moreover, we have $\tilde{q}^{\prime} j f_{2}^{\prime}=\tilde{f}_{2}^{\prime} \tilde{q}^{\prime}$, and thus by the factorisation axiom and the factorisation lemma 5.1 there exist S-denominators $k, \tilde{k}$, T-denominators $r, \tilde{r}$ and a morphism $f_{2}^{\prime \prime}$ in $\mathcal{C}$ with $\tilde{q} j^{\prime}=k r, \tilde{q}^{\prime} j=\tilde{k} \tilde{r}, \tilde{r} f_{2}^{\prime}=f_{2}^{\prime \prime} r, \tilde{f}_{2}^{\prime} k=\tilde{k} f_{2}^{\prime \prime}$. We choose a T-denominator $\tilde{r}^{\prime}$ and a morphism $f_{1}^{\prime \prime}$ in $\mathcal{C}$ with $f_{1}^{\prime \prime} \tilde{r}=\tilde{r}^{\prime} f_{1}^{\prime}$, and we choose an S-denominator $k^{\prime}$ and a morphism $f_{3}^{\prime \prime}$ in $\mathcal{C}$ with $k f_{3}^{\prime \prime}=\tilde{f}_{3}^{\prime} k^{\prime}$. Then we obtain $\tilde{r}^{\prime} f_{1}^{\prime} f_{2}^{\prime}=f_{1}^{\prime \prime} f_{2}^{\prime \prime} r$, $\tilde{j} k f_{3}^{\prime \prime}=f_{3} \tilde{j}^{\prime} k^{\prime}, \tilde{f}_{2}^{\prime} \tilde{f}_{3}^{\prime} k^{\prime}=\tilde{k} f_{2}^{\prime \prime} f_{3}^{\prime \prime}, f_{1}^{\prime \prime} \tilde{r} q=\tilde{r}^{\prime} q^{\prime} f_{1}$, and therefore

$$
\begin{aligned}
& \bar{c}\left(\bar{c}\left(b_{1} \backslash f_{1} / a_{1}, b_{2} \backslash f_{2} / a_{2}\right), b_{3} \backslash f_{3} / a_{3}\right)=\bar{c}\left(q^{\prime} b_{1} \backslash f_{1}^{\prime} f_{2}^{\prime} / a_{2} j^{\prime}, b_{3} \backslash f_{3} / a_{3}\right) \\
& =\tilde{r}^{\prime} q^{\prime} b_{1} \backslash f_{1}^{\prime \prime} f_{2}^{\prime \prime} f_{3}^{\prime \prime} / a_{3} \tilde{j}^{\prime} k^{\prime}=\bar{c}\left(b_{1} \backslash f_{1} / a_{1}, \tilde{q}^{\prime} b_{2} \backslash \tilde{f}_{2}^{\prime} \tilde{f}_{3}^{\prime} / a_{3} \tilde{j}^{\prime}\right) \\
& =\bar{c}\left(b_{1} \backslash f_{1} / a_{1}, \bar{c}\left(b_{2} \backslash f_{2} / a_{2}, b_{3} \backslash f_{3} / a_{3}\right)\right) .
\end{aligned}
$$

Thus $\bar{c}$ is associative.

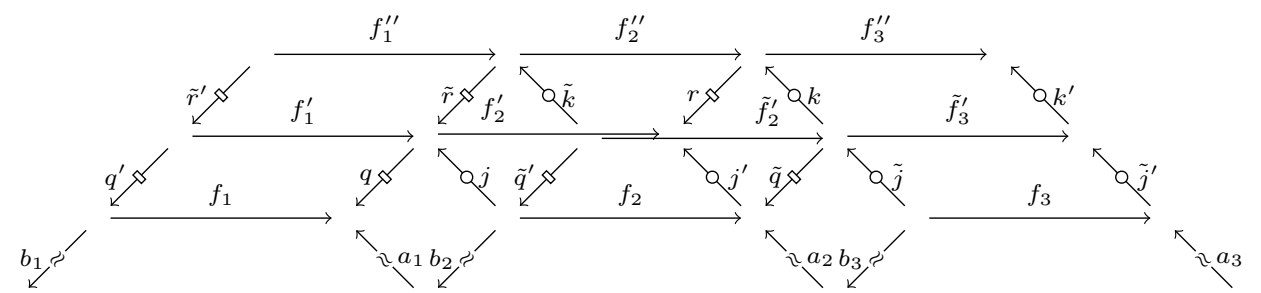

Finally, we suppose given $(b, f, a) \in \operatorname{Arr} A G \mathcal{C}$. We want to show that we have $\bar{c}(b \backslash f / a, e($ Target $b \backslash f / a))=b \backslash f / a$. By the normalisation lemma 4.9 , there exists a normal arrow $(p, g, i) \in \operatorname{Arr} \operatorname{AGC}$ with $(b, f, a) \equiv(p, g, i)$. We obtain

$\bar{c}(b \backslash f / a, e($ Target $b \backslash f / a))=\bar{c}(p \backslash g / i, 1 \backslash 1 / 1)=1 p \backslash g 1 / 1 i=p \backslash g / i=b \backslash f / a$. 
Analogously, we have $\bar{c}(e($ Source $b \backslash f / a), b \backslash f / a)=b \backslash f / a$.
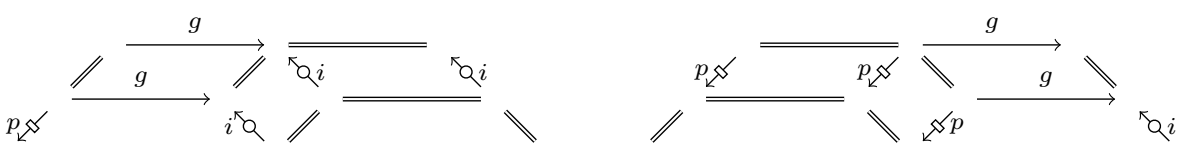

Altogether, $(\mathrm{AGC}) / \equiv$ becomes a category with composition $\left(b_{1} \backslash f_{1} / a_{1}\right)\left(b_{2} \backslash f_{2} / a_{2}\right)=$ $\bar{c}\left(b_{1} \backslash f_{1} / a_{1}, b_{2} \backslash f_{2} / a_{2}\right)$ for $\left(b_{1}, f_{1}, a_{1}\right),\left(b_{2}, f_{2}, a_{2}\right) \in \operatorname{Arr}$ AGC with Target $b_{1} \backslash f_{1} / a_{1}=$ Source $b_{2} \backslash f_{2} / a_{2}$ and identities $1_{X}=e(X)$ for $X \in \mathrm{Ob}(\mathrm{AGC}) / \equiv$.

Definition 5.3 (fraction category). The fraction category of $\mathcal{C}$ is defined to be the category Frac $\mathcal{C}$, whose underlying graph is given by the quotient graph $(\mathrm{AGC}) / \equiv$ and whose composition and identities are given as in proposition 5.2.

Our next aim is to show that the fraction category of a uni-fractionable category is a localisation, which is going to be the second part of our main theorem 5.13.

Remark 5.4. We have

$$
\left(b_{1} \backslash f_{1} / 1\right)\left(1 \backslash f_{2} / a_{2}\right)=b_{1} \backslash f_{1} f_{2} / a_{2}
$$

for all 3 -arrows $\left(b_{1}, f_{1}, 1\right)$ and $\left(1, f_{2}, a_{2}\right)$ in $\mathcal{C}$.

Proof. This follows using the definition of the composition in proposition 5.2.

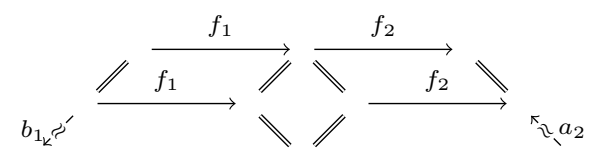

Proposition 5.5 (universal property of the fraction category). The fraction category Frac $\mathcal{C}$ is a localisation of $\mathcal{C}$ with respect to Den $\mathcal{C}$, where the localisation functor loc: $\mathcal{C} \rightarrow$ Frac $\mathcal{C}$ is given on the objects by $\operatorname{loc}(X)=X$ for $X \in \mathrm{Ob} \mathcal{C}$ and on the morphisms by $\operatorname{loc}(f)=1 \backslash f / 1$ for $f \in \operatorname{Mor} \mathcal{C}$. The inverse of $\operatorname{loc}(d)$ for $d \in \operatorname{Den} \mathcal{C}$ is given by $(\operatorname{loc}(d))^{-1}=d \backslash 1 / 1=1 \backslash 1 / d$.

Given a category $\mathcal{D}$ and a functor $F: \mathcal{C} \rightarrow \mathcal{D}$ such that $F d$ is invertible for all $d \in$ Den $\mathcal{C}$, the unique functor $\hat{F}:$ Frac $\mathcal{C} \rightarrow \mathcal{D}$ with $F=\hat{F} \circ$ loc is given by $\hat{F}(b \backslash f / a)=$ $(F b)^{-1}(F f)(F a)^{-1}$.

Proof. We define a graph morphism $L: \mathcal{C} \rightarrow$ Frac $\mathcal{C}$ on the objects by $L X:=X$ for $X \in \mathrm{Ob} \mathcal{C}$ and on the arrows by $L f:=1 \backslash f / 1$ for $f \in \operatorname{Mor} \mathcal{C}$. By remark 5.4, we get

$$
L(f g)=1 \backslash f g / 1=(1 \backslash f / 1)(1 \backslash g / 1)=(L f)(L g)
$$

for all $f, g \in \operatorname{Mor} \mathcal{C}$ with Target $f=$ Source $g$ and

$$
L 1_{X}=1_{X} \backslash 1_{X} / 1_{X}=1_{L X}
$$

for all $X \in \mathrm{Ob} \mathcal{C}$, that is, $L$ is a functor. We want to show that $\operatorname{Frac} \mathcal{C}$ is a localisation of $\mathcal{C}$ with localisation functor $L$.

(Inv) We suppose given $d \in \operatorname{Den} \mathcal{C}$. By remark 5.4, we have

$$
(L d)(1 \backslash 1 / d)=(1 \backslash d / 1)(1 \backslash 1 / d)=1 \backslash d / d=1 \backslash 1 / 1=1
$$

and analogously $(d \backslash 1 / 1)(L d)=1$, that is, $L d$ has a right inverse $1 \backslash 1 / d$ and a left inverse $d \backslash 1 / 1$. But then $L d$ is invertible and the left and the right inverse coincide as 
the unique inverse of $L d$, that is, $(L d)^{-1}=d \backslash 1 / 1=1 \backslash 1 / d$.

(1-uni) We let $\mathcal{D}$ be a category and $F: \mathcal{C} \rightarrow \mathcal{D}$ be a functor such that $F d$ is invertible for all $d \in \operatorname{Den} \mathcal{C}$. For $(b, f, a) \in \operatorname{Arr} \operatorname{AGC}$, we have

$$
\begin{aligned}
\text { Source }\left((F b)^{-1}(F f)(F a)^{-1}\right) & =\operatorname{Source}(F b)^{-1}=\operatorname{Target}(F b)=F(\text { Target } b) \\
& =F(\operatorname{Source}(b, f, a)),
\end{aligned}
$$

and analogously Target $\left((F b)^{-1}(F f)(F a)^{-1}\right)=F$ (Target $\left.(b, f, a)\right)$. Thus there exists a graph morphism $F^{\prime}:$ AGC $\rightarrow \mathcal{D}$ given on the objects by $F^{\prime} X=F X$ for $X \in$ Ob AGC and on the arrows by $F^{\prime}(b, f, a)=(F b)^{-1}(F f)(F a)^{-1}$ for $(b, f, a) \in$ Arr AGC . Moreover, given $(b, f, a) \in \operatorname{Arr} \mathrm{AG} \mathcal{C}$ and $c, c^{\prime} \in \operatorname{Den} \mathcal{C}$ with Target $c^{\prime}=$ Source $b$, Source $c=$ Target $a$, we obtain

$$
\begin{aligned}
F^{\prime}\left(c^{\prime} b, c^{\prime} f c, a c\right) & =\left(F\left(c^{\prime} b\right)\right)^{-1}\left(F\left(c^{\prime} f c\right)\right)(F(a c))^{-1} \\
& =\left(\left(F c^{\prime}\right)(F b)\right)^{-1}\left(\left(F c^{\prime}\right)(F f)(F c)\right)((F a)(F c))^{-1} \\
& =(F b)^{-1}\left(F c^{\prime}\right)^{-1}\left(F c^{\prime}\right)(F f)(F c)(F c)^{-1}(F a)^{-1} \\
& =(F b)^{-1}(F f)(F a)^{-1}=F^{\prime}(b, f, a) .
\end{aligned}
$$

Hence $F^{\prime}$ maps fraction equal 3 -arrows to the same morphism and we obtain an induced graph morphism $\hat{F}:(\mathrm{AGC}) / \equiv \rightarrow \mathcal{D}$ with $F^{\prime}=\hat{F} \circ$ quo.

We want to show that $\hat{F}$ is a functor. Given $\left(b_{1}, f_{1}, a_{1}\right),\left(b_{2}, f_{2}, a_{2}\right) \in \operatorname{Arr}$ AGC $\mathcal{C}$ with Target $\left(b_{1}, f_{1}, a_{1}\right)=$ Source $\left(b_{2}, f_{2}, a_{2}\right)$, we have

$$
\begin{aligned}
& \hat{F}\left(\left(b_{1} \backslash f_{1} / a_{1}\right)\left(b_{2} \backslash f_{2} / a_{2}\right)\right)=\hat{F}\left(q^{\prime} b_{1} \backslash f_{1}^{\prime} f_{2}^{\prime} / a_{2} j^{\prime}\right) \\
& =\left(F\left(q^{\prime} b_{1}\right)\right)^{-1}\left(F\left(f_{1}^{\prime} f_{2}^{\prime}\right)\right)\left(F\left(a_{2} j^{\prime}\right)\right)^{-1}=\left(F b_{1}\right)^{-1}\left(F q^{\prime}\right)^{-1}\left(F f_{1}^{\prime}\right)\left(F f_{2}^{\prime}\right)\left(F j^{\prime}\right)^{-1}\left(F a_{2}\right)^{-1} \\
& =\left(F b_{1}\right)^{-1}\left(F f_{1}\right)(F q)^{-1}(F j)^{-1}\left(F f_{2}\right)\left(F a_{2}\right)^{-1} \\
& =\left(F b_{1}\right)^{-1}\left(F f_{1}\right)\left(F a_{1}\right)^{-1}\left(F b_{2}\right)^{-1}\left(F f_{2}\right)\left(F a_{2}\right)^{-1}=\hat{F}\left(b_{1} \backslash f_{1} / a_{1}\right) \hat{F}\left(b_{2} \backslash f_{2} / a_{2}\right),
\end{aligned}
$$

where $j, j^{\prime}, q, q^{\prime}, f_{1}^{\prime}, f_{2}^{\prime}$ are supposed to be constructed as in proposition 5.2.

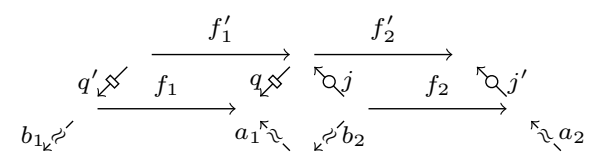

Moreover, we have

$$
\hat{F}\left(1_{X}\right)=\hat{F}\left(1_{X} \backslash 1_{X} / 1_{X}\right)=\left(F 1_{X}\right)^{-1}\left(F 1_{X}\right)\left(F 1_{X}\right)^{-1}=1_{F X}=1_{\hat{F} X}
$$

for $X \in \mathrm{Ob}$ Frac $\mathcal{C}$. This implies that $\hat{F}: \operatorname{Frac} \mathcal{C} \rightarrow \mathcal{D}$ is a functor, given by $\hat{F}(b \backslash f / a)=$ $F^{\prime}(b, f, a)=(F b)^{-1}(F f)(F a)^{-1}$ for $(b, f, a) \in$ Arr AGC . In particular,

$$
\hat{F} L f=\hat{F}(1 \backslash f / 1)=(F 1)^{-1}(F f)(F 1)^{-1}=F f
$$

for all $f \in \operatorname{Mor} \mathcal{C}$, that is, $\hat{F} \circ L=F$.

Conversely, given an arbitrary functor $G: \operatorname{Frac} \mathcal{C} \rightarrow \mathcal{D}$ with $F=G \circ L$, we conclude by remark 5.4 that

$$
\begin{aligned}
G(b \backslash f / a) & =G((b \backslash 1 / 1)(1 \backslash f / 1)(1 \backslash 1 / a))=G\left((L b)^{-1}(L f)(L a)^{-1}\right) \\
& =(G L b)^{-1}(G L f)(G L a)^{-1}=(F b)^{-1}(F f)(F a)^{-1}
\end{aligned}
$$

for $(b, f, a) \in \operatorname{Arr}$ AGC . 
(2-uni) We suppose given a category $\mathcal{D}$ and functors $F, G: \mathcal{C} \rightarrow \mathcal{D}$ such that $F d$ and $G d$ are invertible for all $d \in \operatorname{Den} \mathcal{C}$, and we let $\hat{F}, \hat{G}$ : Frac $\mathcal{C} \rightarrow \mathcal{D}$ be the unique functors with $F=\hat{F} \circ L$ resp. $G=\hat{G} \circ L$. Moreover, we suppose given a transfor-

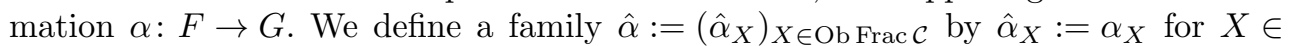
$\mathrm{Ob} \operatorname{Frac} \mathcal{C}=\mathrm{Ob} \mathcal{C}$. Then $\hat{\alpha}_{L X}=\hat{\alpha}_{X}=\alpha_{X}$ for $X \in \mathrm{Ob} \mathcal{C}$. Moreover, $\hat{\alpha}$ is a transformation from $\hat{F}$ to $\hat{G}$ as for every 3 -arrow $(b, f, a): X \leftarrow \tilde{X} \rightarrow \tilde{Y} \leftarrow Y$ in $\mathcal{C}$, we have

$$
\begin{aligned}
& \hat{\alpha}_{X}(\hat{G}(b \backslash f / a))=\alpha_{X}(G b)^{-1}(G f)(G a)^{-1}=(F b)^{-1} \alpha_{\tilde{X}}(G f)(G a)^{-1} \\
& =(F b)^{-1}(F f) \alpha_{\tilde{Y}}(G a)^{-1}=(F b)^{-1}(F f)(F a)^{-1} \alpha_{Y}=(\hat{F}(b \backslash f / a)) \hat{\alpha}_{Y} .
\end{aligned}
$$

Conversely, given an arbitrary transformation $\beta: \hat{F} \rightarrow \hat{G}$ such that $\beta_{L X}=\alpha_{X}$ for all $X \in \mathrm{Ob} \mathcal{C}$, we necessarily have $\beta_{X}=\beta_{L X}=\alpha_{X}$ for all $X \in \mathrm{Ob}$ Frac $\mathcal{C}=\mathrm{Ob} \mathcal{C}$.

Corollary 5.6 (splitting double fractions). For each 3 -arrow $(b, f, a)$ in $\mathcal{C}$, we have

$$
b \backslash f / a=(\operatorname{loc}(b))^{-1} \operatorname{loc}(f)(\operatorname{loc}(a))^{-1} .
$$

Proof. By proposition 5.5, the fraction category Frac $\mathcal{C}$ is a localisation of $\mathcal{C}$ with respect to Den $\mathcal{C}$. In particular, $\operatorname{loc}(d)$ is invertible for all $d \in \operatorname{Den} \mathcal{C}$, and hence there exists a unique functor $\hat{L}: \operatorname{Frac} \mathcal{C} \rightarrow \operatorname{Frac} \mathcal{C}$ with loc $=\hat{L} \circ$ loc, which is given by $\hat{L}(b \backslash f / a)=(\operatorname{loc}(b))^{-1} \operatorname{loc}(f)(\operatorname{loc}(a))^{-1}$ for $(b, f, a) \in \operatorname{Arr}$ AGC . But since loc $=$ $\operatorname{id}_{\text {Frac } \mathcal{C}} \circ$ loc, we must have $\hat{L}=\operatorname{id}_{\text {Frac } \mathcal{C}}$ and therefore the assertion holds.

In the construction of the composition of the fraction category in proposition 5.2, the occurring morphisms $j, j^{\prime}$ were S-denominators, and $q, q^{\prime}$ were T-denominators. We shall now show that it suffices to have a diagram with arbitrary denominators at their places to get the correct composite.

Proposition 5.7. (a) We suppose given 3 -arrows $\left(b_{1}, f_{1}, a_{1}\right),\left(b_{2}, f_{2}, a_{2}\right)$ in $\mathcal{C}$ with Target $\left(b_{1}, f_{1}, a_{1}\right)=$ Source $\left(b_{2}, f_{2}, a_{2}\right)$. Moreover, we suppose given denominators $d$, $d^{\prime}, e, e^{\prime}$ and morphisms $g_{1}, g_{2}$ in $\mathcal{C}$ with $b_{2} a_{1}=d e, g_{1} e=e^{\prime} f_{1}, d g_{2}=f_{2} d^{\prime}$. Then we have $\left(b_{1} \backslash f_{1} / a_{1}\right)\left(b_{2} \backslash f_{2} / a_{2}\right)=e^{\prime} b_{1} \backslash g_{1} g_{2} / a_{2} d^{\prime}$.

(b) Given a 3-arrow $(b, d, a)$ in $\mathcal{C}$ with a denominator $d$, the double fraction $b \backslash d / a$ is invertible in Frac $\mathcal{C}$, and the inverse of $b \backslash d / a$ can be constructed as follows. We choose denominators $d_{1}, d_{1}^{\prime}, d_{2}, d_{2}^{\prime}, a^{\prime}, b^{\prime}$ in $\mathcal{C}$ with $d=d_{1} d_{2}, d_{1} b^{\prime}=b d_{1}^{\prime}, a^{\prime} d_{2}=d_{2}^{\prime} a$. Then we have $(b \backslash d / a)^{-1}=d_{2}^{\prime} \backslash a^{\prime} b^{\prime} / d_{1}^{\prime}$.
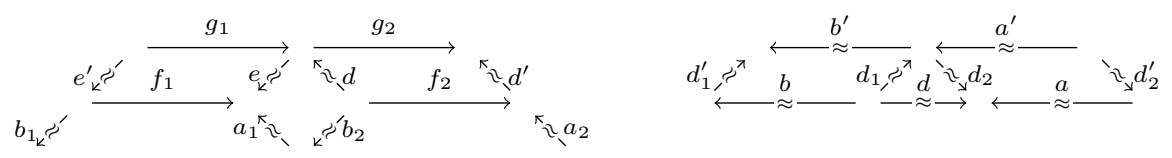

Proof. (a) We compute

$$
\begin{aligned}
& \left(b_{1} \backslash f_{1} / a_{1}\right)\left(b_{2} \backslash f_{2} / a_{2}\right) \\
& =\left(\operatorname{loc}\left(b_{1}\right)\right)^{-1} \operatorname{loc}\left(f_{1}\right)\left(\operatorname{loc}\left(a_{1}\right)\right)^{-1}\left(\operatorname{loc}\left(b_{2}\right)\right)^{-1} \operatorname{loc}\left(f_{2}\right)\left(\operatorname{loc}\left(a_{2}\right)\right)^{-1} \\
& =\left(\operatorname{loc}\left(b_{1}\right)\right)^{-1} \operatorname{loc}\left(f_{1}\right)(\operatorname{loc}(e))^{-1}(\operatorname{loc}(d))^{-1} \operatorname{loc}\left(f_{2}\right)\left(\operatorname{loc}\left(a_{2}\right)\right)^{-1} \\
& =\left(\operatorname{loc}\left(b_{1}\right)\right)^{-1}\left(\operatorname{loc}\left(e^{\prime}\right)\right)^{-1} \operatorname{loc}\left(g_{1}\right) \operatorname{loc}\left(g_{2}\right)\left(\operatorname{loc}\left(d^{\prime}\right)\right)^{-1}\left(\operatorname{loc}\left(a_{2}\right)\right)^{-1} \\
& =\left(\operatorname{loc}\left(e^{\prime} b_{1}\right)\right)^{-1} \operatorname{loc}\left(g_{1} g_{2}\right)\left(\operatorname{loc}\left(a_{2} d^{\prime}\right)\right)^{-1}=e^{\prime} b_{1} \backslash g_{1} g_{2} / a_{2} d^{\prime} .
\end{aligned}
$$


(b) The double fraction $b \backslash d / a=(\operatorname{loc}(b))^{-1} \operatorname{loc}(d)(\operatorname{loc}(a))^{-1}$ is invertible in Frac $\mathcal{C}$ since the localisation functor loc: $\mathcal{C} \rightarrow$ Frac $\mathcal{C}$ maps denominators in $\mathcal{C}$ to isomorphisms in $\operatorname{Frac} \mathcal{C}$.

Given denominators $d_{1}, d_{1}^{\prime}, d_{2}, d_{2}^{\prime}, a^{\prime}, b^{\prime}$ in $\mathcal{C}$ with $d=d_{1} d_{2}, d_{1} b^{\prime}=b d_{1}^{\prime}, a^{\prime} d_{2}=d_{2}^{\prime} a$, we obtain

$$
\begin{aligned}
(b \backslash d / a)^{-1} & =\left((\operatorname{loc}(b))^{-1} \operatorname{loc}(d)(\operatorname{loc}(a))^{-1}\right)^{-1}=\operatorname{loc}(a)(\operatorname{loc}(d))^{-1} \operatorname{loc}(b) \\
& =\operatorname{loc}(a)\left(\operatorname{loc}\left(d_{1} d_{2}\right)\right)^{-1} \operatorname{loc}(b)=\operatorname{loc}(a)\left(\operatorname{loc}\left(d_{2}\right)\right)^{-1}\left(\operatorname{loc}\left(d_{1}\right)\right)^{-1} \operatorname{loc}(b) \\
& =\left(\operatorname{loc}\left(d_{2}^{\prime}\right)\right)^{-1} \operatorname{loc}\left(a^{\prime}\right) \operatorname{loc}\left(b^{\prime}\right)\left(\operatorname{loc}\left(d_{1}^{\prime}\right)\right)^{-1} \\
& =\left(\operatorname{loc}\left(d_{2}^{\prime}\right)\right)^{-1} \operatorname{loc}\left(a^{\prime} b^{\prime}\right)\left(\operatorname{loc}\left(d_{1}^{\prime}\right)\right)^{-1}=d_{2}^{\prime} \backslash a^{\prime} b^{\prime} / d_{1}^{\prime} .
\end{aligned}
$$

Proposition 5.8. Given a uni-fractionable category $\mathcal{D}$ and a denominator preserving functor $F: \mathcal{C} \rightarrow \mathcal{D}$, there exists a unique induced functor

\section{Frac $F:$ Frac $\mathcal{C} \rightarrow \operatorname{Frac} \mathcal{D}$}

with $\operatorname{loc}^{\operatorname{Frac} \mathcal{D}} \circ F=(\operatorname{Frac} F) \circ \operatorname{loc}^{\operatorname{Frac} \mathcal{C}}$. It is given on the objects by $(\operatorname{Frac} F) X=$ $F X$ for $X \in \mathrm{Ob}$ Frac $\mathcal{C}$ and on the morphisms by $(\operatorname{Frac} F)(b \backslash f / a)=F b \backslash F f / F a$ for $(b, f, a) \in \operatorname{Arr} \mathrm{AGC}$.

Proof. Since $F$ preserves denominators and $\operatorname{loc}^{\operatorname{Frac} \mathcal{D}}$ maps denominators in $\mathcal{D}$ to isomorphisms in Frac $\mathcal{D}$, the composite $\operatorname{loc}^{\operatorname{Frac} \mathcal{D}} \circ F$ maps denominators in $\mathcal{C}$ to isomorphisms in $\operatorname{Frac} \mathcal{D}$. By the universal property of $\operatorname{Frac} \mathcal{C}$, there exists a unique functor Frac $F:$ Frac $\mathcal{C} \rightarrow \operatorname{Frac} \mathcal{D}$ with $\operatorname{loc}^{\operatorname{Frac} \mathcal{D}} \circ F=(\operatorname{Frac} F) \circ \operatorname{loc}^{\operatorname{Frac} \mathcal{C}}$. It follows that

$$
(\operatorname{Frac} F) X=(\operatorname{Frac} F) \operatorname{loc}(X)=\operatorname{loc}(F X)=F X
$$

for $X \in \mathrm{Ob} \mathcal{C}$ as well as

$$
\begin{aligned}
(\operatorname{Frac} F)(b \backslash f / a) & =(\operatorname{Frac} F)\left((\operatorname{loc}(b))^{-1} \operatorname{loc}(f)(\operatorname{loc}(a))^{-1}\right) \\
& =((\operatorname{Frac} F) \operatorname{loc}(b))^{-1}((\operatorname{Frac} F) \operatorname{loc}(f))((\operatorname{Frac} F) \operatorname{loc}(a))^{-1} \\
& =(\operatorname{loc}(F b))^{-1} \operatorname{loc}(F f)(\operatorname{loc}(F a))^{-1}=F b \backslash F f / F a
\end{aligned}
$$

for $(b, f, a) \in \operatorname{Arr} \mathrm{AGC}$.

Here is another elementary property of the fraction category, which will be needed in proposition 5.15, where we deal with coproducts.

Proposition 5.9. We suppose given morphisms $\varphi_{1}$ and $\varphi_{2}$ in Frac $\mathcal{C}$.

(a) If Source $\varphi_{1}=$ Source $\varphi_{2}$, then there exist normal 3 -arrows $\left(p, f_{1}, i_{1}\right),\left(p, f_{2}, i_{2}\right)$ in $\mathcal{C}$ with $\varphi_{1}=p \backslash f_{1} / i_{1}$ and $\varphi_{2}=p \backslash f_{2} / i_{2}$.

(b) If Source $\varphi_{1}=$ Source $\varphi_{2}$ and Target $\varphi_{1}=$ Target $\varphi_{2}$, then there exist normal 3-arrows $\left(p, f_{1}, i\right),\left(p, f_{2}, i\right)$ in $\mathcal{C}$ with $\varphi_{1}=p \backslash f_{1} / i$ and $\varphi_{2}=p \backslash f_{2} / i$.

Proof. (a) We choose 3 -arrows $\left(b_{k}, g_{k}, a_{k}\right)$ with $\varphi_{k}=b_{k} \backslash g_{k} / a_{k}$ for $k \in\{1,2\}$. By the normalisation lemma 4.9 , there exist normal 3 -arrows $\left(p_{k}, g_{k}^{\prime}, i_{k}\right)$ in $\mathcal{C}$ with $\left(b_{k}, g_{k}, a_{k}\right)$ $\equiv\left(p_{k}, g_{k}^{\prime}, i_{k}\right)$ for $k \in\{1,2\}$, that is, with $\varphi_{k}=b_{k} \backslash g_{k} / a_{k}=p_{k} \backslash g_{k}^{\prime} / i_{k}$ for $k \in\{1,2\}$.

There exist a T-denominator $p_{2}^{\prime}$ and a morphism $p_{1}^{\prime}$ in $\mathcal{C}$ with $p_{2}^{\prime} p_{1}=p_{1}^{\prime} p_{2}$. We define $p:=p_{2}^{\prime} p_{1}=p_{1}^{\prime} p_{2}, f_{1}:=p_{2}^{\prime} g_{1}^{\prime}, f_{2}:=p_{1}^{\prime} g_{2}^{\prime}$. By multiplicativity, $p=p_{2}^{\prime} p_{1}$ is a T-denominator in $\mathcal{C}$, and we have $\varphi_{1}=p_{1} \backslash g_{1}^{\prime} / i_{1}=p_{2}^{\prime} p_{1} \backslash p_{2}^{\prime} g_{1}^{\prime} / i_{1}=p \backslash f_{1} / i_{1}$ and analogously $\varphi_{2}=p \backslash f_{2} / i_{2}$. 
(b) This is proven similarly to (a).
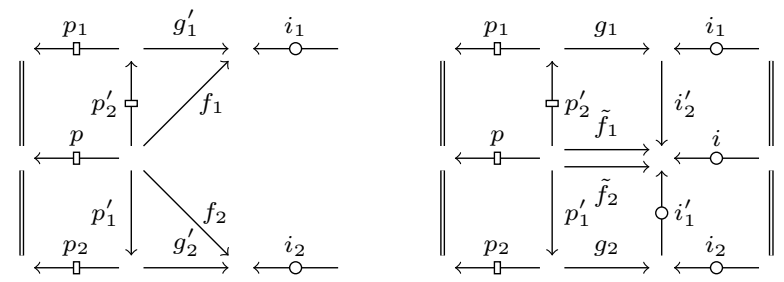

Proposition 5.10 (cf. [4, sec. 36.4]). The denominator set in $\mathcal{C}$ is saturated if and only if it is weakly saturated.

Proof. Since saturatedness always implies weak saturatedness, it suffices to show that if Den $\mathcal{C}$ is weakly saturated, then it is already saturated. So we suppose that Den $\mathcal{C}$ is weakly saturated and we suppose given a morphism $f$ in $\mathcal{C}$ such that $\operatorname{loc}(f)$ is invertible in Frac $\mathcal{C}$. We let $(p, g, i)$ be a normal 3 -arrow in $\mathcal{C}$ with $(\operatorname{loc}(f))^{-1}=p \backslash g / i$. Moreover, we choose a T-denominator $p^{\prime}$ and a morphism $f^{\prime}$ in $\mathcal{C}$ with $f^{\prime} p=p^{\prime} f$, and we choose an S-denominator $i^{\prime}$ and a morphism $f^{\prime \prime}$ in $\mathcal{C}$ with $i f^{\prime \prime}=f i^{\prime}$.

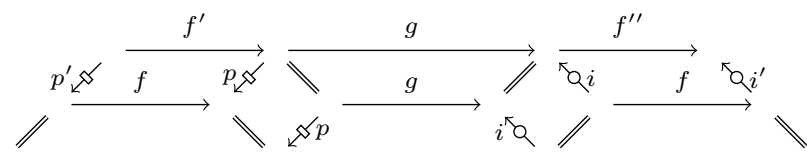

Then we have $1 \backslash 1 / 1=(1 \backslash f / 1)(p \backslash g / i)=p^{\prime} \backslash f^{\prime} g / i$ and $1 \backslash 1 / 1=(p \backslash g / i)(1 \backslash f / 1)=$ $p \backslash g f^{\prime \prime} / i^{\prime}$. By remark 4.5, the morphisms $f^{\prime} g$ and $g f^{\prime \prime}$ must be denominators. Now (2 of 6) implies that $f^{\prime}$ and thus $f$ is a denominator. Hence Den $\mathcal{C}$ is saturated.

Now we come to the last part of the main theorem of this article, that is, we want to show that $\mathcal{C}$ admits a 3 -arrow calculus. It can be found in proposition 5.12. The key step of its proof is treated in the following lemma.

Lemma 5.11 (flipping lemma). Given 3-arrows $\left(b_{1}, f_{1}, a_{1}\right),\left(b_{2}, f_{2}, a_{2}\right),\left(v_{1}, h_{1}, u_{1}\right)$, $\left(v_{2}, h_{2}, u_{2}\right)$, morphisms $g_{1}, g_{1}^{\prime}, g_{1}^{\prime \prime}, g_{2}, g_{2}^{\prime}, g_{2}^{\prime \prime}$, denominators $d$, e, an S-denominator $i_{2}$ and a T-denominator $p_{1}$ in $\mathcal{C}$, fitting into the commutative diagram in $\mathcal{C}$ on the left, there exist 3 -arrows $\left(\tilde{b}_{1}, \tilde{f}_{1}, \tilde{a}_{1}\right),\left(\tilde{b}_{2}, \tilde{f}_{2}, \tilde{a}_{2}\right)$ and normal 3 -arrows $\left(\tilde{p}_{1}, \tilde{g}_{1}, \tilde{i}_{1}\right)$, $\left(\tilde{p}_{2}, \tilde{g}_{2}, \tilde{i}_{2}\right)$, fitting into the commutative diagram in $\mathcal{C}$ on the right.
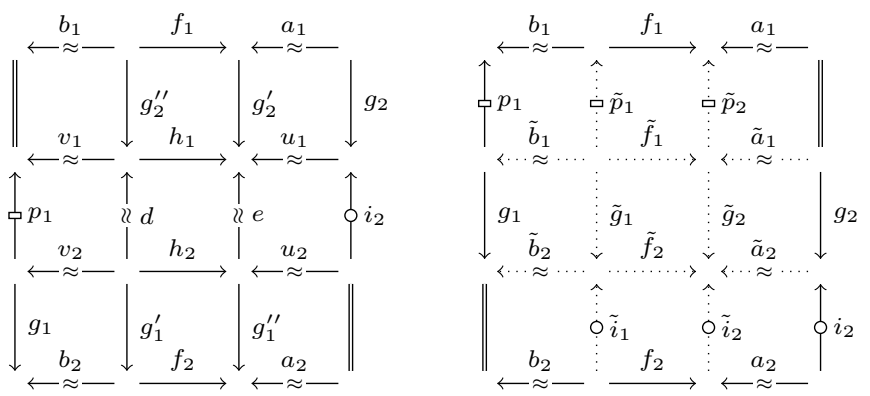
Proof. By the factorisation axiom and the factorisation lemma 5.1, there exist S-denominators $j_{1}, \tilde{j}_{2}$, T-denominators $q_{1}, \tilde{q}_{2}$ and morphisms $b, \tilde{a}$ in $\mathcal{C}$ with $d=j_{1} q_{1}$, $e=\tilde{j}_{2} \tilde{q}_{2}, q_{1} v_{1}=b p_{1}, v_{2}=j_{1} b, u_{1}=\tilde{a} \tilde{q}_{2}, u_{2} \tilde{j}_{2}=i_{2} \tilde{a}$. Moreover, by the factorisation lemma 5.1, there exist an S-denominator $j_{2}$, a T-denominator $q_{2}$, a morphism $f$ and a denominator $\tilde{a}^{\prime}$ in $\mathcal{C}$ with $e=j_{2} q_{2}, q_{1} h_{1}=f q_{2}, j_{1} f=h_{2} j_{2}, \tilde{q}_{2}=\tilde{a}^{\prime} q_{2}, j_{2}=\tilde{j}_{2} \tilde{a}^{\prime}$.
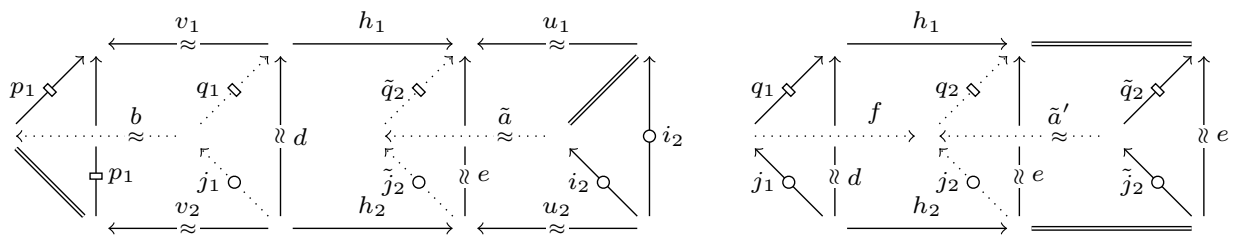

We set $a:=\tilde{a} \tilde{a}^{\prime}$ and obtain $u_{1}=a q_{2}$ and $u_{2} j_{2}=i_{2} a$. Next, we choose weak pullback rectangles

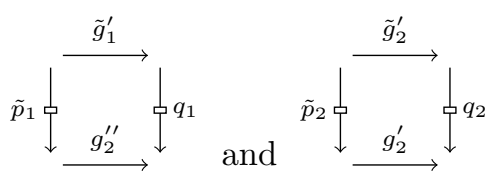

in $\mathcal{C}$ such that $\tilde{p}_{1}$ and $\tilde{p}_{2}$ are T-denominators, and we choose weak pushout rectangles

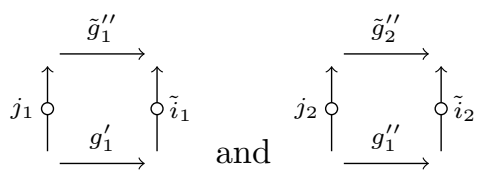

in $\mathcal{C}$ such that $\tilde{i}_{1}$ and $\tilde{i}_{2}$ are S-denominators. We obtain induced morphisms $\tilde{b}_{1}, \tilde{f}_{1}, \tilde{a}_{1}$ on the weak pullbacks, that is, with $\tilde{p}_{1} b_{1}=\tilde{b}_{1} p_{1}, \tilde{b}_{1}=\tilde{g}_{1}^{\prime} b, \tilde{p}_{1} f_{1}=\tilde{f}_{1} \tilde{p}_{2}, \tilde{f}_{1} \tilde{g}_{2}^{\prime}=\tilde{g}_{1}^{\prime} f$, $a_{1}=\tilde{a}_{1} \tilde{p}_{2}, \tilde{a}_{1} \tilde{g}_{2}^{\prime}=g_{2} a$, and induced morphisms $\tilde{b}_{2}, \tilde{f}_{2}, \tilde{a}_{2}$ on the weak pushouts, that is, with $b g_{1}=\tilde{g}_{1}^{\prime \prime} \tilde{b}_{2}, \tilde{i}_{1} \tilde{b}_{2}=b_{2}, f \tilde{g}_{2}^{\prime \prime}=\tilde{g}_{1}^{\prime \prime} \tilde{f}_{2}, \tilde{i}_{1} \tilde{f}_{2}=f_{2} \tilde{i}_{2}, a \tilde{g}_{2}^{\prime \prime}=\tilde{a}_{2}, i_{2} \tilde{a}_{2}=a_{2} \tilde{i}_{2}$.

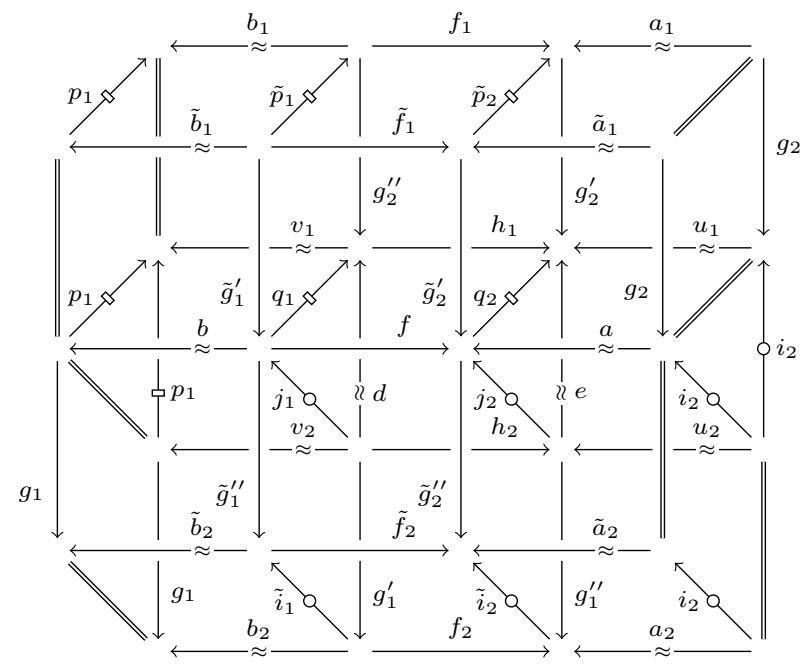


Setting $\tilde{g}_{1}:=\tilde{g}_{1}^{\prime} \tilde{g}_{1}^{\prime \prime}$ and $\tilde{g}_{2}:=\tilde{g}_{2}^{\prime} \tilde{g}_{2}^{\prime \prime}$ yields $\tilde{b}_{1} g_{1}=\tilde{g}_{1} \tilde{b}_{2}, \tilde{f}_{1} \tilde{g}_{2}=\tilde{g}_{1} \tilde{f}_{2}, \tilde{a}_{1} \tilde{g}_{2}=g_{2} \tilde{a}_{2}$. Moreover, $\tilde{a}_{1}, \tilde{a}_{2}, \tilde{b}_{1}, \tilde{b}_{2}$ are denominators in $\mathcal{C}$ by semi-saturatedness.

Proposition 5.12 (3-arrow calculus, cf. [4, sec. 36.3]). (a) Given 3-arrows $\left(b_{1}, f_{1}, a_{1}\right)$ and $\left(b_{2}, f_{2}, a_{2}\right)$ in $\mathcal{C}$, we have $b_{1} \backslash f_{1} / a_{1}=b_{2} \backslash f_{2} / a_{2}$ in Frac $\mathcal{C}$ if and only if there exist 3 -arrows $\left(\tilde{b}_{1}, \tilde{f}_{1}, \tilde{a}_{1}\right),\left(\tilde{b}_{2}, \tilde{f}_{2}, \tilde{a}_{2}\right)$ and normal 3 -arrows $\left(p_{1}, d_{1}, i_{1}\right),\left(p_{2}, d_{2}, i_{2}\right)$ with denominators $d_{1}, d_{2}$, fitting into the commutative diagram in $\mathcal{C}$ on the left.

If $\left(b_{1}, f_{1}, a_{1}\right),\left(b_{2}, f_{2}, a_{2}\right)$ are normal 3 -arrows, then $\left(\tilde{b}_{1}, \tilde{f}_{1}, \tilde{a}_{1}\right),\left(\tilde{b}_{2}, \tilde{f}_{2}, \tilde{a}_{2}\right)$ can be chosen to be normal, too.

(b) Given 3-arrows $\left(b_{1}, f_{1}, a_{1}\right),\left(b_{2}, f_{2}, a_{2}\right)$ and normal 3-arrows $\left(p_{1}, g_{1}, i_{1}\right)$, $\left(p_{2}, g_{2}, i_{2}\right)$ in $\mathcal{C}$, we have $\left(b_{1} \backslash f_{1} / a_{1}\right)\left(p_{2} \backslash g_{2} / i_{2}\right)=\left(p_{1} \backslash g_{1} / i_{1}\right)\left(b_{2} \backslash f_{2} / a_{2}\right)$ in Frac $\mathcal{C}$ if and only if there exist 3 -arrows $\left(\tilde{b}_{1}, \tilde{f}_{1}, \tilde{a}_{1}\right),\left(\tilde{b}_{2}, \tilde{f}_{2}, \tilde{a}_{2}\right)$ and normal 3 -arrows $\left(\tilde{p}_{1}, \tilde{g}_{1}, \tilde{i}_{1}\right)$, $\left(\tilde{p}_{2}, \tilde{g}_{2}, \tilde{i}_{2}\right)$, fitting into the commutative diagram in $\mathcal{C}$ on the right.
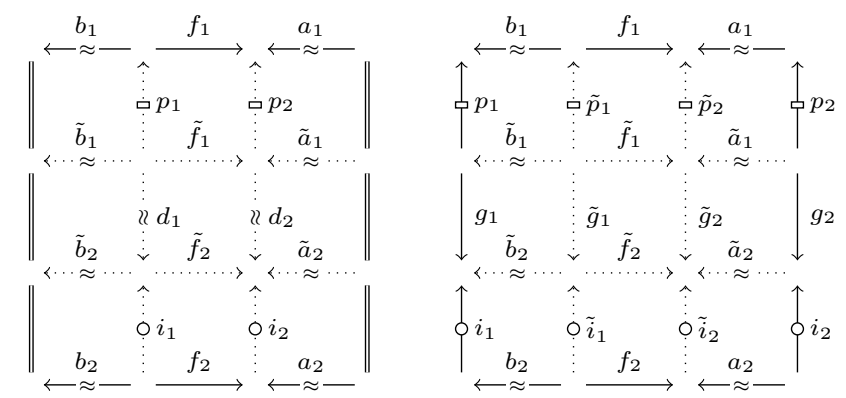

Proof. (a) If we have a commutative diagram as stated, then we have

$$
\left(b_{1}, f_{1}, a_{1}\right) \equiv\left(\tilde{b}_{1}, \tilde{f}_{1}, \tilde{a}_{1}\right) \equiv\left(\tilde{b}_{2}, \tilde{f}_{2}, \tilde{a}_{2}\right) \equiv\left(b_{2}, f_{2}, a_{2}\right)
$$

and thus $b_{1} \backslash f_{1} / a_{1}=b_{2} \backslash f_{2} / a_{2}$ in Frac $\mathcal{C}$.

So we suppose conversely that $b_{1} \backslash f_{1} / a_{1}=b_{2} \backslash f_{2} / a_{2}$ in $\operatorname{Frac} \mathcal{C}$, that is, we suppose that $\left(b_{1}, f_{1}, a_{1}\right) \equiv\left(b_{2}, f_{2}, a_{2}\right)$ in AGC . By remark $4.4(\mathrm{~b})$, there exist $n \in \mathbb{N}_{0}$, $\left(v_{l}, h_{l}, u_{l}\right) \in \operatorname{Arr} \mathrm{AGC}$ for $l \in[0,2 n+1], c_{l}, c_{l}^{\prime} \in \operatorname{Mor} \mathcal{C}$ for $l \in[0, n], w_{l}, w_{l}^{\prime}$ for $l \in$ $[0, n-1]$, with $\left(v_{0}, h_{0}, u_{0}\right)=\left(b_{1}, f_{1}, a_{1}\right),\left(v_{2 n+1}, h_{2 n+1}, u_{2 n+1}\right)=\left(b_{2}, f_{2}, a_{2}\right)$ and $v_{2 l}=$ $c_{l} v_{2 l+1}, h_{2 l} c_{l}^{\prime}=c_{l} h_{2 l+1}, u_{2 l} c_{l}^{\prime}=u_{2 l+1}$ for $l \in[0, n]$ and $v_{2 l+2}=w_{l} v_{2 l+1}, w_{l} h_{2 l+1}=$ $h_{2 l+2} w_{l}^{\prime}, u_{2 l+2} w_{l}^{\prime}=u_{2 l+1}$ for $l \in[0, n-1]$.

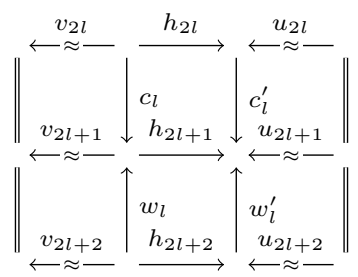

By semi-saturatedness, $c_{l}$ and $c_{l}^{\prime}$ are denominators for all $l \in[0, n]$ and $w_{l}, w_{l}^{\prime}$ are denominators for all $l \in[0, n-1]$. Using the flipping lemma 5.11 and induction on $n \in \mathbb{N}_{0}$ yields the first assertion.

Now let us suppose that $\left(b_{1}, f_{1}, a_{1}\right)$ and $\left(b_{2}, f_{2}, a_{2}\right)$ are normal 3 -arrows. By multiplicativity, $\tilde{b}_{1}=p_{1} b_{1}$ is a T-denominator and $\tilde{a}_{2}=a_{2} i_{2}$ is an S-denominator in $\mathcal{C}$. We 
choose S-denominators $j_{1}, j_{2}$ and T-denominators $q_{1}, q_{2}$ with $\tilde{a}_{1}=j_{1} q_{1}$ and $\tilde{b}_{2}=j_{2} q_{2}$. Moreover, we choose a T-denominator $q_{1}^{\prime}$ and a morphism $\tilde{f}_{1}^{\prime}$ in $\mathcal{C}$ with $\tilde{f}_{1}^{\prime} q_{1}=q_{1}^{\prime} \tilde{f}_{1}$, and we choose an S-denominator $j_{2}^{\prime}$ and a morphism $\tilde{f}_{2}^{\prime}$ in $\mathcal{C}$ with $j_{2} \tilde{f}_{2}^{\prime}=\tilde{f}_{2} j_{2}^{\prime}$.

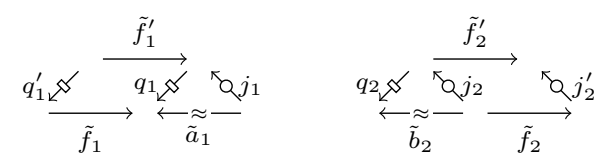

By multiplicativity, $q_{1}^{\prime} \tilde{b}_{1}=q_{1}^{\prime} p_{1} b_{1}, q_{1}^{\prime} p_{1}, q_{1} p_{2}$ are T-denominators and $\tilde{a}_{2} j_{2}^{\prime}=a_{2} i_{2} j_{2}^{\prime}$, $i_{1} j_{2}, i_{2} j_{2}^{\prime}$ are $\mathrm{S}$-denominators in $\mathcal{C}$. Now the diagram

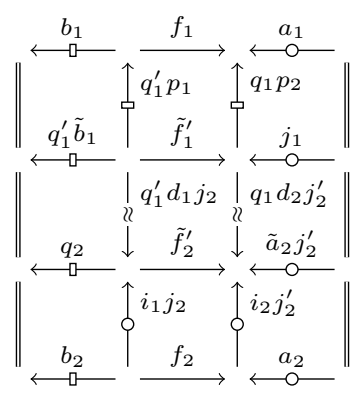

commutes and $\left(q_{1}^{\prime} \tilde{b}_{1}, \tilde{f}_{1}^{\prime}, j_{1}\right),\left(q_{2}, \tilde{f}_{2}^{\prime}, \tilde{a}_{2} j_{2}^{\prime}\right),\left(q_{1}^{\prime} p_{1}, q_{1}^{\prime} d_{1} j_{2}, i_{1} j_{2}\right),\left(q_{1} p_{2}, q_{1} d_{2} j_{2}^{\prime}, i_{2} j_{2}^{\prime}\right)$ are normal 3-arrows.

(b) If we have a commutative diagram as stated, then proposition 5.7(a) implies that

$$
\begin{aligned}
\left(b_{1} \backslash f_{1} / a_{1}\right)\left(p_{2} \backslash g_{2} / i_{2}\right) & =\tilde{p}_{1} b_{1} \backslash \tilde{f}_{1} \tilde{g}_{2} / i_{2} \tilde{a}_{2}=\tilde{b}_{1} p_{1} \backslash \tilde{g}_{1} \tilde{f}_{2} / a_{2} \tilde{i}_{2} \\
& =\left(p_{1} \backslash g_{1} / i_{1}\right)\left(b_{2} \backslash f_{2} / a_{2}\right) .
\end{aligned}
$$

So we suppose conversely that $\left(b_{1} \backslash f_{1} / a_{1}\right)\left(p_{2} \backslash g_{2} / i_{2}\right)=\left(p_{1} \backslash g_{1} / i_{1}\right)\left(b_{2} \backslash f_{2} / a_{2}\right)$. We construct $\left(b_{1} \backslash f_{1} / a_{1}\right)\left(p_{2} \backslash g_{2} / i_{2}\right)=q^{\prime} b_{1} \backslash f_{1}^{\prime} g_{2}^{\prime} / i_{2} j^{\prime}$ and $\left(p_{1} \backslash g_{1} / i_{1}\right)\left(b_{2} \backslash f_{2} / a_{2}\right)=$ $\tilde{q}^{\prime} p_{1} \backslash g_{1}^{\prime} f_{2}^{\prime} / a_{2} \tilde{j}^{\prime}$ as in proposition 5.2.

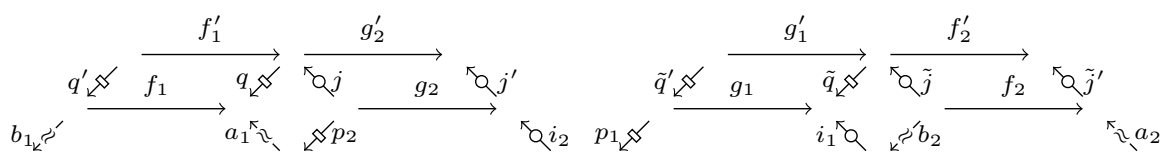

By(a), as $\left(b_{1} \backslash f_{1} / a_{1}\right)\left(p_{2} \backslash g_{2} / i_{2}\right)=\left(p_{1} \backslash g_{1} / i_{1}\right)\left(b_{2} \backslash f_{2} / a_{2}\right)$ and therefore $q^{\prime} b_{1} \backslash f_{1}^{\prime} g_{2}^{\prime} / i_{2} j^{\prime}=$ $\tilde{q}^{\prime} p_{1} \backslash g_{1}^{\prime} f_{2}^{\prime} / a_{2} \tilde{j}^{\prime}$, there exist 3 -arrows $\left(v_{1}, h_{1}, u_{1}\right),\left(v_{2}, h_{2}, u_{2}\right)$ and normal 3 -arrows $\left(r_{1}, d_{1}, k_{1}\right),\left(r_{2}, d_{2}, k_{2}\right)$ in $\mathcal{C}$ with denominators $d_{1}, d_{2}$, fitting into the commutative diagram displayed below on the left. Altogether, we obtain the commutative diagram displayed below on the right. Applying the flipping lemma 5.11 twice and composing yields the assertion. 

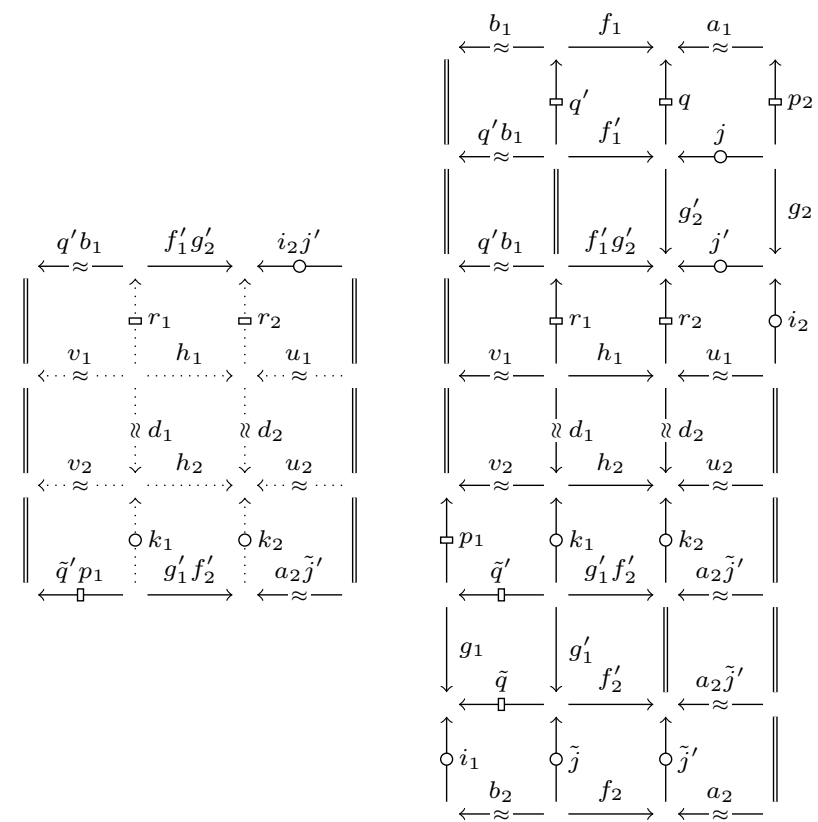

Altogether, we have proven the following main theorem of this article. Recall that $\mathcal{C}$ is supposed to be a uni-fractionable category, see definition 3.1.

Theorem 5.13. The fraction category Frac $\mathcal{C}$ fulfills the following properties.

(a) The object set of Frac $\mathcal{C}$ is the object set of $\mathcal{C}$. The morphism set of Frac $\mathcal{C}$ consists of double fractions, that is, equivalence classes of 3-arrows with respect to fraction equality, where a 3 -arrow $(b, f, a)$ is a diagram

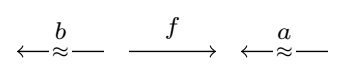

in $\mathcal{C}$ with denominators $a$ and $b$. For every 3 -arrow $(b, f, a)$ in $\mathcal{C}$, source and target of the double fraction $b \backslash f / a$ are given by Source $b \backslash f / a=$ Target $b$ and Target $b \backslash f / a=$ Source $a$. Given 3-arrows $\left(b_{1}, f_{1}, a_{1}\right)$ and $\left(b_{2}, f_{2}, a_{2}\right)$ in $\mathcal{C}$ with Target $b_{1} \backslash f_{1} / a_{1}=$ Source $b_{2} \backslash f_{2} / a_{2}$, the composite of the double fractions can be constructed as follows: One chooses denominators $d, d^{\prime}, e, e^{\prime}$ and morphisms $g_{1}, g_{2}$ in $\mathcal{C}$ with $b_{2} a_{1}=d e$, $g_{1} e=e^{\prime} f_{1}, d g_{2}=f_{2} d^{\prime}$. Then $\left(b_{1} \backslash f_{1} / a_{1}\right)\left(b_{2} \backslash f_{2} / a_{2}\right)=e^{\prime} b_{1} \backslash g_{1} g_{2} / a_{2} d^{\prime}$.

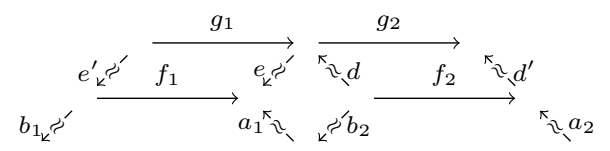

The identity of an object $X$ in Frac $\mathcal{C}$ is given by $1_{X}=1_{X} \backslash 1_{X} / 1_{X}$.

(b) The fraction category $\operatorname{Frac} \mathcal{C}$ is a localisation of $\mathcal{C}$ with respect to Den $\mathcal{C}$, where the localisation functor loc: $\mathcal{C} \rightarrow \operatorname{Frac} \mathcal{C}$ is given on the objects by $\operatorname{loc}(X)=X$ for $X \in \mathrm{Ob} \mathcal{C}$ and on the morphisms by $\operatorname{loc}(f)=1 \backslash f / 1$ for $f \in \operatorname{Mor} \mathcal{C}$. The inverse of $\operatorname{loc}(d)$ for $d \in \operatorname{Den} \mathcal{C}$ is given by $(\operatorname{loc}(d))^{-1}=d \backslash 1 / 1=1 \backslash 1 / d$. 
Given a category $\mathcal{D}$ and a functor $F: \mathcal{C} \rightarrow \mathcal{D}$ such that $F d$ is invertible for all $d \in$ Den $\mathcal{C}$, the unique functor $\hat{F}:$ Frac $\mathcal{C} \rightarrow \mathcal{D}$ with $F=\hat{F} \circ$ loc is given by $\hat{F}(b \backslash f / a)=$ $(F b)^{-1}(F f)(F a)^{-1}$.

(c) Given 3-arrows $\left(b_{1}, f_{1}, a_{1}\right),\left(b_{2}, f_{2}, a_{2}\right)$ and normal 3-arrows $\left(p_{1}, g_{1}, i_{1}\right)$, $\left(p_{2}, g_{2}, i_{2}\right)$ in $\mathcal{C}$, we have $\left(b_{1} \backslash f_{1} / a_{1}\right)\left(p_{2} \backslash g_{2} / i_{2}\right)=\left(p_{1} \backslash g_{1} / i_{1}\right)\left(b_{2} \backslash f_{2} / a_{2}\right)$ in Frac $\mathcal{C}$ if and only if there exist 3 -arrows $\left(\tilde{b}_{1}, \tilde{f}_{1}, \tilde{a}_{1}\right),\left(\tilde{b}_{2}, \tilde{f}_{2}, \tilde{a}_{2}\right)$ and normal 3 -arrows $\left(\tilde{p}_{1}, \tilde{g}_{1}, \tilde{i}_{1}\right)$, $\left(\tilde{p}_{2}, \tilde{g}_{2}, \tilde{i}_{2}\right)$, fitting into the following commutative diagram in $\mathcal{C}$.

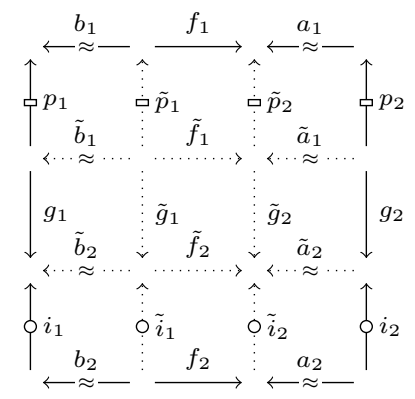

Proof. This follows from propositions 5.2 and 5.7(a), proposition 5.5 and proposition $5.12(\mathrm{~b})$.

As a consequence of 3-arrow calculus, we get the following criterion. For a related 2-arrow version of this result, cf. [15, ch. 1, §2, th. 4-2] and [8, III.2.10].

Proposition 5.14. We suppose given a uni-fractionable category $\mathcal{U}$ such that $\mathcal{U}$ is a full subcategory of $\mathcal{C}$ and $\operatorname{Den} \mathcal{U}=(\operatorname{Den} \mathcal{C}) \cap($ Mor $\mathcal{U})$. We suppose that for every object $X$ in $\mathcal{C}$, there exist an object $\tilde{X}$ in $\mathcal{U}$ and a denominator $d: \tilde{X} \rightarrow X$ in $\mathcal{C}$. We suppose that for every $S$-denominator $i: U \rightarrow \tilde{U}$ with $U$ in $\mathcal{U}$, it follows that $\tilde{U}$ is in $\mathcal{U}$. Then the inclusion functor inc: $\mathcal{U} \rightarrow \mathcal{C}$ induces an equivalence Frac inc: $\operatorname{Frac} \mathcal{U} \rightarrow$ Frac $\mathcal{C}$.

Proof. To show that Fracinc is an equivalence of categories, we will verify that Frac inc is full, faithful and dense. Since for every $X \in \mathrm{Ob} \mathcal{C}$ there exist $\tilde{X} \in \mathrm{Ob} \mathcal{U}$ and a denominator $d: \tilde{X} \rightarrow X$ in $\mathcal{C}$, we have $X \cong \tilde{X}=($ Fracinc $) \tilde{X}$ in Frac $\mathcal{C}$. Hence Frac inc is dense. To prove that Frac inc is full and faithful, we have to show that the $\operatorname{map} \operatorname{Frac} \mathcal{U}(U, V) \rightarrow \operatorname{Frac} \mathcal{C}(U, V), \varphi \mapsto($ Fracinc $) \varphi$ is bijective for $U, V \in \mathrm{Ob} \mathcal{U}$.

To show surjectivity, we suppose given a morphism $\psi \in \operatorname{Frac} \mathcal{C}(U, V)$ and a normal 3-arrow $(p, f, i): U \leftarrow X \rightarrow Y \leftarrow V$ in $\mathcal{C}$ with $\psi=p \backslash f / i$. Since $i$ is an S-denominator and $V$ is an object in $\mathcal{U}$, it follows that $Y$ is an object in $\mathcal{U}$. Moreover, there exists an object $\tilde{X}$ in $\mathcal{U}$ and a denominator $d: \tilde{X} \rightarrow X$.

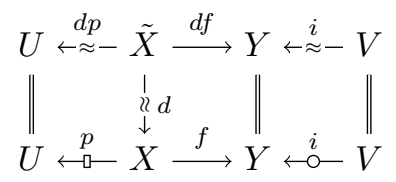

It follows that $(p, f, i) \equiv(d p, d f, i)$, and as $(d p, d f, i)$ is a 3 -arrow in $\mathcal{U}$, we have $\psi=$ $p \backslash f / i=d p \backslash d f / i=($ Fracinc $)(d p \backslash d f / i)$. Thus the map $\operatorname{Frac} \mathcal{U}(U, V) \rightarrow \operatorname{Frac} \mathcal{C}(U, V)$, $\varphi \mapsto($ Frac inc $) \varphi$ is surjective. 
To show injectivity, we suppose given $\varphi_{1}, \varphi_{2} \in$ Frac $\mathcal{U}(U, V)$ with (Fracinc) $\varphi_{1}=$ (Fracinc) $\varphi_{2}$. We choose normal 3-arrows $\left(p_{1}, f_{1}, i_{1}\right): U \leftarrow U_{1} \rightarrow V_{1} \leftarrow V$ and $\left(p_{2}, f_{2}, i_{2}\right): U \leftarrow U_{2} \rightarrow V_{2} \leftarrow V$ in $\mathcal{U}$ with $\varphi_{1}=p_{1} \backslash f_{1} / i_{1}$ and $\varphi_{2}=p_{2} \backslash f_{2} / i_{2}$. By proposition $5.12(\mathrm{a})$, there exist normal 3 -arrows $\left(\tilde{p}_{1}, \tilde{f}_{1}, \tilde{i}_{1}\right): U \leftarrow X_{1} \rightarrow Y_{1} \leftarrow V$, $\left(\tilde{p}_{2}, \tilde{f}_{2}, \tilde{i}_{2}\right): U \leftarrow X_{2} \rightarrow Y_{2} \leftarrow V,\left(q_{1}, d_{1}, j_{1}\right): U_{1} \leftarrow X_{1} \rightarrow X_{2} \leftarrow U_{2},\left(q_{2}, d_{2}, j_{2}\right): V_{1} \leftarrow$ $Y_{1} \rightarrow Y_{2} \leftarrow V_{2}$ in $\mathcal{C}$ with denominators $d_{1}, d_{2}$, fitting into a commutative diagram as displayed below on the left. Since $\tilde{i}_{1}$ resp. $j_{1}$ resp. $j_{2}$ is an S-denominator and $V$ resp. $U_{2}$ resp. $V_{2}$ is an object in $\mathcal{U}$, it follows that $Y_{1}$ resp. $X_{2}$ resp. $Y_{2}$ is an object in $\mathcal{U}$. Moreover, there exists an object $\tilde{X}_{1}$ in $\mathcal{U}$ and a denominator $d: \tilde{X}_{1} \rightarrow X_{1}$ in $\mathcal{C}$. Thus we obtain the commutative diagram displayed below on the right, in which all objects - and hence all morphisms - are in $\mathcal{U}$, and where $d \tilde{p}_{1}$ is a denominator by multiplicativity. It follows that $\varphi_{1}=p_{1} \backslash f_{1} / i_{1}=p_{2} \backslash f_{2} / i_{2}=\varphi_{2}$ in Frac $\mathcal{U}$. Thus the map $\operatorname{Frac}_{\mathcal{U}}(U, V) \rightarrow \operatorname{Frac}_{\mathcal{C}}(U, V), \varphi \mapsto($ Frac inc $) \varphi$ is injective.

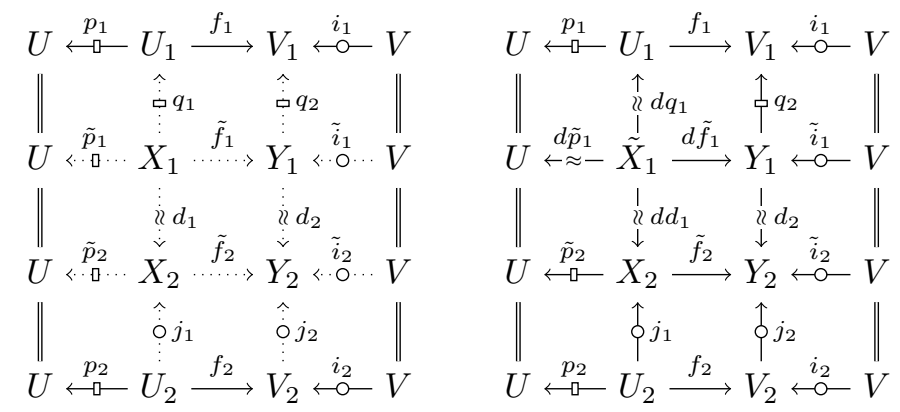

Some of our examples of uni-fractionable categories in section 6 have finite coproducts, so it is a natural question to ask whether these are preserved when passing to the fraction category.

Proposition 5.15. We suppose that $\mathcal{C}$ admits finite coproducts.

(a) If Den $\mathcal{C}$ is closed under finite coproducts, then the fraction category Frac $\mathcal{C}$ admits finite coproducts and the localisation functor loc: $\mathcal{C} \rightarrow$ Frac $\mathcal{C}$ preserves finite coproducts. In this case, we have $\operatorname{ini}_{\operatorname{loc}(X)} \operatorname{loc}\left(\mathrm{i}_{)}\right) \operatorname{loc}\left(\operatorname{ini}_{X}^{i}\right): \operatorname{loc}(\mathrm{i}) \rightarrow \operatorname{loc}(X)$ for $X \in \mathrm{ObC}$, and we have

$$
\left(\begin{array}{l}
b_{1} \backslash f_{1} / a \\
b_{2} \backslash f_{2} / a
\end{array}\right)^{\operatorname{loc}\left(X_{1} \amalg X_{2}\right)}=\left(b_{1} \amalg b_{2}\right) \backslash\left(\begin{array}{l}
f_{1} \\
f_{2}
\end{array}\right)^{\tilde{X}_{1} \amalg \tilde{X}_{2}} / a: \operatorname{loc}\left(X_{1} \amalg X_{2}\right) \rightarrow \operatorname{loc}(Y)
$$

for 3-arrows $\left(b_{1}, f_{1}, a\right): X_{1} \leftarrow \tilde{X}_{1} \rightarrow \tilde{Y} \leftarrow Y,\left(b_{2}, f_{2}, a\right): X_{2} \leftarrow \tilde{X}_{2} \rightarrow \tilde{Y} \leftarrow Y$ in $\mathcal{C}$.

(b) If Den $\mathcal{C}$ is saturated and the localisation functor loc: $\mathcal{C} \rightarrow$ Frac $\mathcal{C}$ preserves finite coproducts, then Den $\mathcal{C}$ is closed under finite coproducts.

Proof. (a) We suppose that Den $\mathcal{C}$ is closed under finite coproducts. Moreover, we suppose given $X \in \mathrm{ObC}$. Then $\operatorname{loc}\left(\mathrm{ini}_{X}^{\mathrm{i}}\right)$ is a morphism from $\operatorname{loc}(\mathrm{i})$ to $\operatorname{loc}(X)$. So let us suppose given an arbitrary morphism $\varphi: \operatorname{loc}(\mathrm{i}) \rightarrow X$ in $\operatorname{Frac} \mathcal{C}$, and we let $(b, f, a): \mathrm{i} \leftarrow$ $I \rightarrow \tilde{X} \leftarrow X$ be a 3 -arrow in $\mathcal{C}$ with $\varphi=b \backslash f / a$. By the universal property of $i$, we have $\operatorname{ini}_{I}^{\mathrm{i}} b=1_{\mathrm{i}}$ and $\operatorname{ini}_{I}^{\mathrm{i}} f=\operatorname{ini}_{\tilde{X}}^{\mathrm{i}}=\operatorname{ini}_{X}^{\mathrm{i}} a$, and therefore

$$
\varphi=b \backslash f / a=\operatorname{ini}_{I}^{\mathrm{i}} b \backslash \operatorname{ini}_{I}^{\mathrm{i}} f / a=1_{\mathrm{i}} \backslash \operatorname{ini}_{X}^{\mathrm{i}} / 1=\operatorname{loc}\left(\mathrm{ini}_{X}^{\mathrm{i}}\right) .
$$


Hence $\operatorname{loc}(i)$ is an initial object in $\operatorname{Frac} \mathcal{C}$ with $\operatorname{ini}_{\operatorname{loc}(X)}^{\operatorname{loc}(i)}=\operatorname{loc}\left(\operatorname{ini}_{X}^{i}\right)$ for $X \in \operatorname{Ob} \mathcal{C}$.

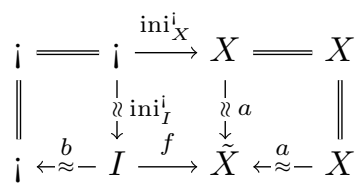

Next, we suppose given morphisms $\varphi_{1}: X_{1} \rightarrow Y$ and $\varphi_{2}: X_{2} \rightarrow Y$ in Frac $\mathcal{C}$. By proposition 5.9, there exist 3-arrows $\left(b_{k}, f_{k}, a\right): X_{k} \leftarrow \tilde{X}_{k} \rightarrow \tilde{Y} \leftarrow Y$ in $\mathcal{C}$ with $\varphi_{k}=$ $b_{k} \backslash f_{k} / a$ for $k \in\{1,2\}$. As $b_{1} \amalg b_{2}$ is a denominator in $\mathcal{C}$ by assumption, we have the 3-arrow $\left(b_{1} \amalg b_{2},\left(\begin{array}{l}f_{1} \\ f_{2}\end{array}\right), a\right)$ in $\mathcal{C}$. Moreover, since $\mathrm{emb}_{k}^{\tilde{X}_{1} \amalg \tilde{X}_{2}}\left(b_{1} \amalg b_{2}\right)=b_{k} \mathrm{emb}_{k}^{X_{1} \amalg X_{2}}$, we have

$$
\begin{aligned}
\operatorname{loc}\left(\operatorname{emb}_{k}^{X_{1} \amalg X_{2}}\right)\left(\left(b_{1} \amalg b_{2}\right) \backslash\left(\begin{array}{c}
f_{1} \\
f_{2}
\end{array}\right)^{\tilde{X}_{1} \amalg \tilde{X}_{2}} / a\right) & =b_{k} \backslash \operatorname{emb}_{k}^{\tilde{X}_{1} \amalg \tilde{X}_{2}}\left(\begin{array}{l}
f_{1} \\
f_{2}
\end{array}\right)^{\tilde{X}_{1} \amalg \tilde{X}_{2}} / a \\
& =b_{k} \backslash f_{k} / a=\varphi_{k}
\end{aligned}
$$

for $k \in\{1,2\}$.

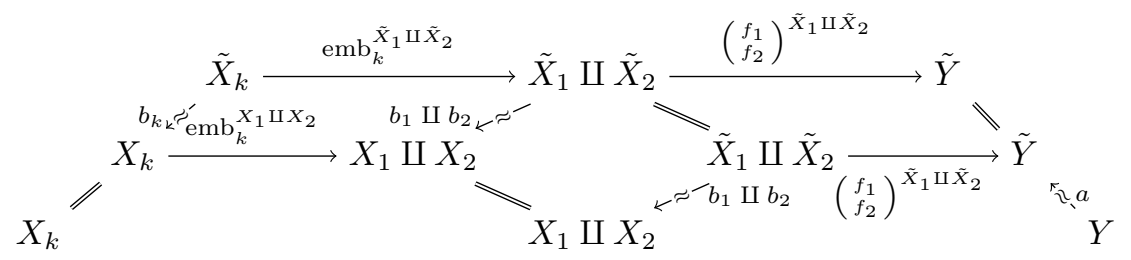

Conversely, we suppose given morphisms $\varphi, \varphi^{\prime}: \operatorname{loc}\left(X_{1} \amalg X_{2}\right) \rightarrow \operatorname{loc}(Y)$ in $\operatorname{Frac} \mathcal{C}$ such that $\operatorname{loc}\left(\mathrm{emb}_{k}^{X_{1} \amalg X_{2}}\right) \varphi=\operatorname{loc}\left(\operatorname{emb}_{k}^{X_{1} \amalg X_{2}}\right) \varphi^{\prime}=\varphi_{k}$ for $k \in\{1,2\}$. Then by proposition 5.9, there exist normal 3-arrows $(p, f, i),\left(p, f^{\prime}, i\right): X_{1} \amalg X_{2} \leftarrow \tilde{X} \rightarrow \tilde{Y} \leftarrow Y$ in $\mathcal{C}$ with $\varphi=p \backslash f / i$ and $\varphi^{\prime}=p \backslash f^{\prime} / i$. For $k \in\{1,2\}$, we choose a T-denominator $p_{k}: \tilde{X}_{k} \rightarrow$ $X_{k}$ and a morphism $e_{k}: \tilde{X}_{k} \rightarrow \tilde{X}$ in $\mathcal{C}$ with $p_{k} \mathrm{emb}_{k}^{X_{1} \amalg X_{2}}=e_{k} p$. We get

$$
\varphi_{k}=\operatorname{loc}\left(\mathrm{emb}_{k}^{X_{1} \amalg X_{2}}\right) \varphi=\operatorname{loc}\left(\mathrm{emb}_{k}^{X_{1} \amalg X_{2}}\right)(p \backslash f / i)=p_{k} \backslash e_{k} f / i
$$

for $k \in\{1,2\}$.

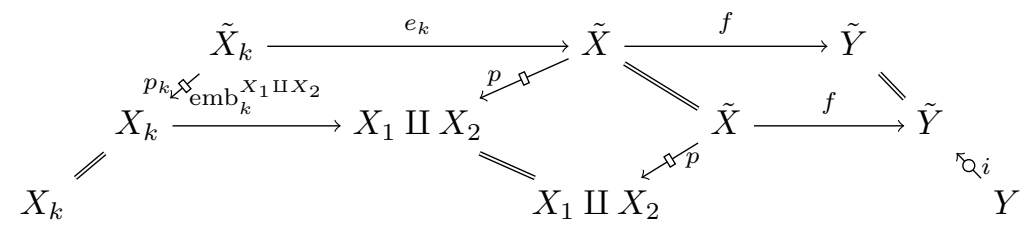

Analogously, we have $\varphi_{k}=p_{k} \backslash e_{k} f^{\prime} / i$ and hence $\operatorname{loc}\left(e_{k} f\right)=\operatorname{loc}\left(e_{k} f^{\prime}\right)$ for $k \in\{1,2\}$. By proposition 5.12(a), there exist normal 3-arrows $\left(\tilde{p}_{k}, \tilde{f}_{k}, \tilde{i}_{k}\right),\left(\tilde{p}_{k}^{\prime}, \tilde{f}_{k}^{\prime}, \tilde{i}_{k}^{\prime}\right),\left(q_{k}, d_{k}, j_{k}\right)$, $\left(\tilde{q}_{k}, \tilde{d}_{k}, \tilde{j}_{k}\right)$ in $\mathcal{C}$ with denominators $d_{k}, \tilde{d}_{k}$ for $k \in\{1,2\}$, fitting into the following commutative diagrams in $\mathcal{C}$. 


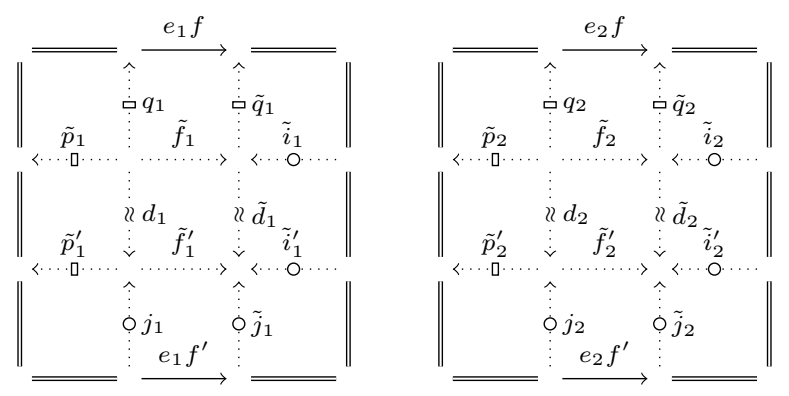

We let

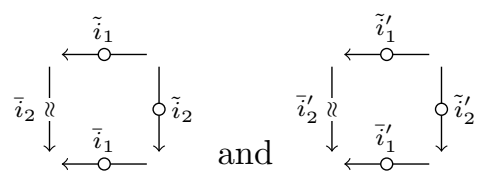

be weak pushout rectangles in $\mathcal{C}$ such that $\bar{i}_{1}$ and $\bar{i}_{1}^{\prime}$ are S-denominators, so that we obtain morphisms $q, d, j$ such that the diagram displayed below on the left commutes. Using coproducts, we obtain the commutative diagram displayed below on the right.

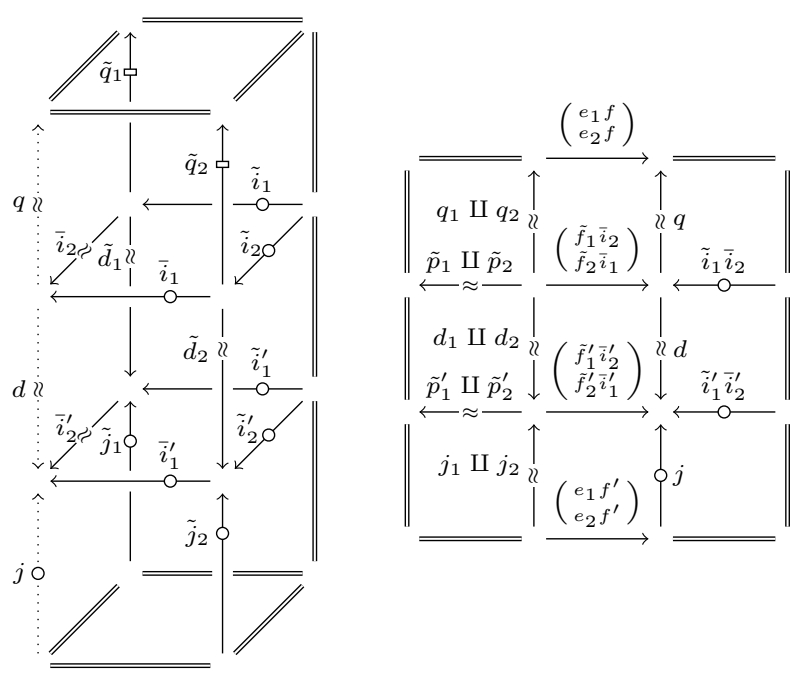

We finally have

$$
\operatorname{loc}\left(\left(\begin{array}{c}
e_{1} \\
e_{2}
\end{array}\right)\right) \operatorname{loc}(f)=\operatorname{loc}\left(\left(\begin{array}{c}
e_{1} f \\
e_{2} f
\end{array}\right)\right)=\operatorname{loc}\left(\left(\begin{array}{c}
e_{1} f^{\prime} \\
e_{2} f^{\prime}
\end{array}\right)\right)=\operatorname{loc}\left(\left(\begin{array}{c}
e_{1} \\
e_{2}
\end{array}\right)\right) \operatorname{loc}\left(f^{\prime}\right) .
$$

On the other hand,

$$
\left(\begin{array}{c}
e_{1} \\
e_{2}
\end{array}\right) p=\left(\begin{array}{c}
e_{1} p \\
e_{2} p
\end{array}\right)=\left(\begin{array}{c}
p_{1} \mathrm{emb}_{1}^{X_{1} \amalg X_{2}} \\
p_{2} \mathrm{emb}_{2}^{X_{1} \amalg X_{2}}
\end{array}\right)=p_{1} \amalg p_{2}
$$

implies that $\left(\begin{array}{l}e_{1} \\ e_{2}\end{array}\right)$ is a denominator in $\mathcal{C}$ by semi-saturatedness, so we have loc $(f)=$ $\operatorname{loc}\left(f^{\prime}\right)$ and therefore $\varphi=p \backslash f / i=p \backslash f^{\prime} / i=\varphi^{\prime}$. 
Altogether, $\operatorname{loc}\left(X_{1} \amalg X_{2}\right)$ is a coproduct of $\operatorname{loc}\left(X_{1}\right)$ and loc $\left(X_{2}\right)$ with embeddings $\mathrm{emb}_{k}^{\operatorname{loc}\left(X_{1} \amalg X_{2}\right)}=\operatorname{loc}\left(\mathrm{emb}_{k}^{X_{1} \amalg X_{2}}\right)$ for $k \in\{1,2\}$.

(b) We suppose that Den $\mathcal{C}$ is saturated and that loc preserves finite coproducts. Moreover, we suppose given denominators $d_{1}: X_{1} \rightarrow Y_{1}$ and $d_{2}: X_{2} \rightarrow Y_{2}$ in $\mathcal{C}$. Then we have

$$
\begin{aligned}
\operatorname{loc}\left(d_{k}\right) \operatorname{emb}_{k}^{\operatorname{loc}\left(Y_{1} \amalg Y_{2}\right)} & =\operatorname{loc}\left(d_{k}\right) \operatorname{loc}\left(\operatorname{emb}_{k}^{Y_{1} \amalg Y_{2}}\right)=\operatorname{loc}\left(d_{k} \operatorname{emb}_{k}^{Y_{1} \amalg Y_{2}}\right) \\
& =\operatorname{loc}\left(\operatorname{emb}_{k}^{X_{1} \amalg X_{2}}\left(d_{1} \amalg d_{2}\right)\right)=\operatorname{loc}\left(\operatorname{emb}_{k}^{X_{1} \amalg X_{2}}\right) \operatorname{loc}\left(d_{1} \amalg d_{2}\right) \\
& =\operatorname{emb}_{k}^{\operatorname{loc}\left(X_{1} \amalg X_{2}\right)} \operatorname{loc}\left(d_{1} \amalg d_{2}\right) .
\end{aligned}
$$

Since $d_{1}$ and $d_{2}$ are denominators, $\operatorname{loc}\left(d_{1}\right)$ and $\operatorname{loc}\left(d_{2}\right)$ are isomorphisms. But then $\operatorname{loc}\left(d_{1} \amalg d_{2}\right)$ is also an isomorphism and hence $d_{1} \amalg d_{2}$ is a denominator since Den $\mathcal{C}$ is saturated.

\section{Applications}

\section{Quillen model categories}

Given a Quillen model category $\mathcal{M}[\mathbf{1 3}$, ch. I, $\S 1$, def. 1], we denote by $\operatorname{Cof}(\mathcal{M})$ resp. $\operatorname{Fib}(\mathcal{M})$ resp. $\operatorname{Bif}(\mathcal{M})$ the full subcategory of cofibrant resp. fibrant resp. bifibrant (that is, cofibrant and fibrant) objects.

Example 6.1. Given a Quillen model category $\mathcal{M}$, the categories $\mathcal{M}$, $\operatorname{Cof}(\mathcal{M})$, $\operatorname{Fib}(\mathcal{M}), \operatorname{Bif}(\mathcal{M})$ carry the structure of uni-fractionable categories, where

$$
\begin{aligned}
\operatorname{Den} \mathcal{C} & =\{w \in \operatorname{Mor} \mathcal{C} \mid w \text { is a weak equivalence }\}, \\
\text { SDen } \mathcal{C} & =\{i \in \operatorname{Mor} \mathcal{C} \mid i \text { is an acyclic cofibration }\}, \\
\text { TDen } \mathcal{C} & =\{p \in \operatorname{Mor} \mathcal{C} \mid p \text { is an acyclic fibration }\}
\end{aligned}
$$

for $\mathcal{C} \in\{\mathcal{M}, \operatorname{Cof}(\mathcal{M}), \operatorname{Fib}(\mathcal{M}), \operatorname{Bif}(\mathcal{M})\}$. In particular, the homotopy category Ho $\mathcal{M}$ is isomorphic to Frac $\mathcal{M}$. If $\mathcal{M}$ is a closed Quillen model category, then Den $\mathcal{C}$ is saturated in $\mathcal{C}$ for $\mathcal{C} \in\{\mathcal{M}, \operatorname{Cof}(\mathcal{M}), \operatorname{Fib}(\mathcal{M}), \operatorname{Bif}(\mathcal{M})\}$. The localisation functor loc: $\mathcal{C} \rightarrow$ Frac $\mathcal{C}$ preserves finite coproducts for $\mathcal{C} \in\{\operatorname{Cof}(\mathcal{M}), \operatorname{Bif}(\mathcal{M})\}$ and finite products for $\mathcal{C} \in\{\boldsymbol{F i b}(\mathcal{M}), \operatorname{Bif}(\mathcal{M})\} .\left({ }^{5}\right)$

Proof. (a) We consider $\mathcal{M}$ and verify the axioms of a uni-fractionable category.

(Cat) By definition of a Quillen model category, weak equivalences, cofibrations and fibrations are closed under composition and contain all isomorphisms. Hence in particular weak equivalences, acyclic cofibrations and acyclic fibrations are closed under composition and contain all identities.

(2 of 3) This holds by definition of a Quillen model category.

\footnotetext{
${ }^{5}$ In general, the localisation functor loc: $\mathcal{M} \rightarrow$ Frac $\mathcal{M}$ does not preserve finite coproducts or finite products since the set of denominators in a closed Quillen model category need not be closed under finite (co)products. A counterexample is provided by $(\mathbb{Z} / 4 \downarrow \bmod (\mathbb{Z} / 4))$, cf. $[\mathbf{6}$, rem. 3.11], as considered in [5, ex.]: The coproduct of $2:(\mathbb{Z} / 4,1) \rightarrow(\mathbb{Z} / 4,2)$ with itself is given by $(20):(\mathbb{Z} / 4,1) \rightarrow(\mathbb{Z} / 4 \oplus \mathbb{Z} / 2,(20))$; the former is a weak equivalence, but the latter is not since $\mathbb{Z} / 4$ is a bijective object and $\mathbb{Z} / 4 \oplus \mathbb{Z} / 2$ is not a bijective object in $\bmod (\mathbb{Z} / 4)$.
} 
(WU) We suppose given an acyclic cofibration $i: X \rightarrow X^{\prime}$ and a morphism $f: X \rightarrow Y$ in $\mathcal{M}$, and we let

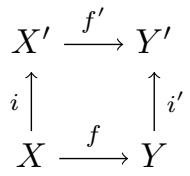

be a pushout rectangle in $\mathcal{C}$. Then $i^{\prime}$ is an acyclic cofibration.

The other assertion follows by duality.

(Fac) Since every morphism decomposes into a composite of a cofibration followed by an acyclic fibration, the assertion follows by semi-saturatedness.

Altogether, $\mathcal{M}$ becomes a uni-fractionable category with

$$
\begin{aligned}
\text { Den } \mathcal{M} & =\{w \in \operatorname{Mor} \mathcal{M} \mid w \text { is a weak equivalence }\}, \\
\text { SDen } \mathcal{M} & =\{i \in \operatorname{Mor} \mathcal{M} \mid i \text { is an acyclic cofibration }\}, \\
\text { TDen } \mathcal{M} & =\{p \in \operatorname{Mor} \mathcal{M} \mid p \text { is an acyclic fibration }\}
\end{aligned}
$$

The assertion on the saturatedness of Den $\mathcal{M}$ is proven in [13, ch. I, $\S 5$, prop. 1].

(b) We consider $\operatorname{Cof}(\mathcal{M})$ and have to verify the axioms of a uni-fractionable category. Since (Cat) and (2 of 3) hold for $\mathcal{M}$ by (a), they hold in particular for $\operatorname{Cof}(\mathcal{M})$.

(WU) We suppose given an acyclic cofibration $i: X \rightarrow X^{\prime}$ and a morphism $f: X \rightarrow Y$ in $\operatorname{Cof}(\mathcal{M})$, and we let

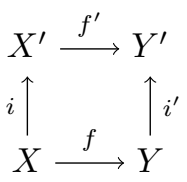

be a pushout rectangle in $\mathcal{C}$. Then $i^{\prime}$ is an acyclic cofibration, and since $Y$ is cofibrant and $i^{\prime}$ is in particular a cofibration, it follows that $Y^{\prime}$ is also cofibrant.

Now we suppose given an acyclic fibration $p: Y^{\prime} \rightarrow Y$ and a morphism $f: X \rightarrow Y$ in $\operatorname{Cof}(\mathcal{M})$, and we let

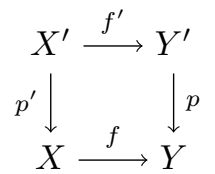

be a pullback rectangle in $\mathcal{C}$. Then $p^{\prime}$ is an acyclic fibration. We consider a strong cofibrant approximation of $X^{\prime}$, that is, we let $\tilde{X}^{\prime}$ be a cofibrant object together with an acyclic fibration $q: \tilde{X}^{\prime} \rightarrow X^{\prime}$. The composite $q p^{\prime}$ is an acyclic fibration by multiplicativity. We will show that

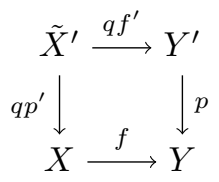

is a weak pullback of $f$ along $p$. To this end, we suppose given an object $T \in$ 
Ob $\operatorname{Cof}(\mathcal{M})$ and morphisms $s: T \rightarrow X, t: T \rightarrow Y^{\prime}$ with $s f=t p$. By the universal property of $X^{\prime}$, there exists a (unique) morphism $u: T \rightarrow X^{\prime}$ such that $u p^{\prime}=s$ and $u f^{\prime}=t$. Moreover, as $T$ is cofibrant and $q$ is an acyclic fibration, there exists a lift $\hat{u}: T \rightarrow \tilde{X}^{\prime}$ such that $u=\hat{u} q$. Now we have $\hat{u} q p^{\prime}=u p^{\prime}=s$ and $\hat{u} q f^{\prime}=u f^{\prime}=t$.
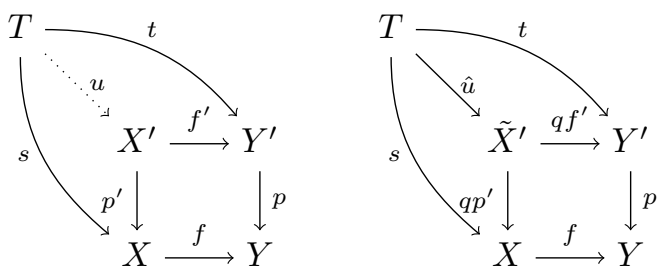

(Fac) We let $w: X \rightarrow Y$ be a weak equivalence in $\operatorname{Cof}(\mathcal{M})$. Then there exists an acyclic cofibration $i: X \rightarrow Z$ and an acyclic fibration $p: Z \rightarrow Y$ in $\mathcal{M}$ with $w=i p$. But since $X$ is cofibrant and $i$ is a cofibration, $Z$ is cofibrant, too.

Altogether, $\operatorname{Cof}(\mathcal{M})$ becomes a uni-fractionable category with

$$
\begin{aligned}
\operatorname{Den} \operatorname{Cof}(\mathcal{M}) & =\{w \in \operatorname{Mor} \operatorname{Cof}(\mathcal{M}) \mid w \text { is a weak equivalence }\}, \\
\operatorname{SDen} \operatorname{Cof}(\mathcal{M}) & =\{i \in \operatorname{Mor} \operatorname{Cof}(\mathcal{M}) \mid i \text { is an acyclic cofibration }\}, \\
\text { TDen } \operatorname{Cof}(\mathcal{M}) & =\{p \in \operatorname{Mor} \operatorname{Cof}(\mathcal{M}) \mid p \text { is an acyclic fibration }\} .
\end{aligned}
$$

The assertion on the saturatedness of $\operatorname{Den} \operatorname{Cof}(\mathcal{M})$ follows from (a) since if $\operatorname{loc}^{\operatorname{Frac} \operatorname{Cof}(\mathcal{M})}(f)$ is an isomorphism, then also $\operatorname{loc}^{\operatorname{Frac} \mathcal{M}}(f)$ is an isomorphism. The fact that the localisation functor loc: $\operatorname{Cof}(\mathcal{M}) \rightarrow \operatorname{Frac} \operatorname{Cof}(\mathcal{M})$ preserves finite coproducts follows from the gluing lemma [10, lem. 7.4], cf. also [9, ch. II, lem. 8.8], and proposition $5.15(\mathrm{a})$.

(c) The proof for $\operatorname{Fib}(\mathcal{M})$ is dual to (b).

(d) The proof for $\operatorname{Bif}(\mathcal{M})$ is a combination of (b) and (c).

As an application of our abstract machinery, we obtain the following part of Quillen's homotopy category theorem [13, ch. I, $\S 1$, th. 1]. Given a Quillen model category $\mathcal{M}$, we (re-)define the homotopy category of $\mathcal{C} \in\{\mathcal{M}, \operatorname{Cof}(\mathcal{M}), \operatorname{Fib}(\mathcal{M})$, $\operatorname{Bif}(\mathcal{M})\}$ by $\operatorname{Ho} \mathcal{C}:=$ Frac $\mathcal{C}$, using the uni-fractionable category structures from the preceding example.

Example 6.2. We suppose given a Quillen model category $\mathcal{M}$. The commutative diagram of inclusion functors displayed below on the left induces the commutative diagram of equivalences displayed below on the right.
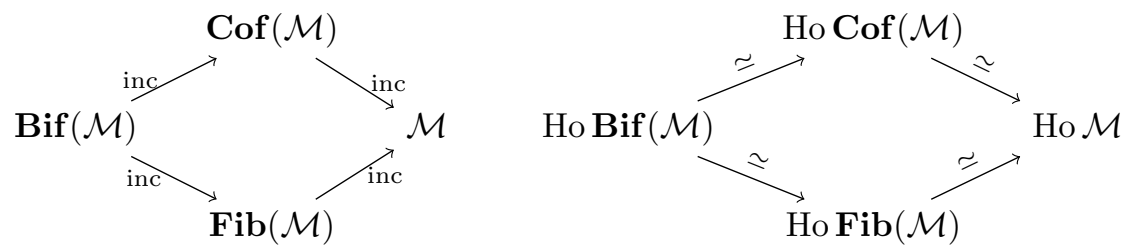

In particular, $\operatorname{Ho} \operatorname{Bif}(\mathcal{M}) \simeq \operatorname{Ho} \mathcal{M}$.

Proof. This follows using proposition 5.14 for inc: $\operatorname{Cof}(\mathcal{M}) \rightarrow \mathcal{M}$ and inc: $\operatorname{Bif}(\mathcal{M})$ $\rightarrow \operatorname{Fib}(\mathcal{M})$, and its dual for inc: $\boldsymbol{F i b}(\mathcal{M}) \rightarrow \mathcal{M}$ and inc: $\operatorname{Bif}(\mathcal{M}) \rightarrow \operatorname{Cof}(\mathcal{M})$. 
The proof of Quillen's homotopy category theorem, which states in particular that the homotopy category Ho $\mathcal{M}$ is equivalent to the quotient category $\operatorname{Bif}(\mathcal{M}) / \sim$, where $\sim$ denotes the homotopy congruence, can now be completed as in $[\mathbf{1 1}$, cor. 1.2.9] by showing that $\operatorname{Bif}(\mathcal{M}) / \sim$ fulfills the universal property of a localisation, which is essentially a corollary of Whitehead's theorem [11, prop. 1.2.8].

\section{Derivable categories}

Recall that a derivable category in the sense of Cisinski [2, sec. 2.25] consists of the same data as a Quillen model category, that is, a category $\mathcal{C}$ together with three distinguished subsets of morphisms, called cofibrations, fibrations and weak equivalences, subject to the following axioms, where (co)fibrant objects and acyclic (co)fibrations are defined as in the Quillen model category case: The set of weak equivalences is supposed to be semi-saturated. The set of cofibrations is supposed to be closed under (binary) composition. There exists an initial object in $\mathcal{C}$, which is supposed to be cofibrant. The set of cofibrant objects is supposed to be closed under isomorphisms. The set of cofibrations between cofibrant objects and the subset of acyclic cofibrations therein are supposed to be stable under pushouts along morphisms between cofibrant objects. Every morphism with cofibrant source object factors into a cofibration followed by a weak equivalence. And dually for the fibrations and fibrant objects.

For homotopical algebra in derivable categories, cf. also the manuscript of RĂDULESCU-BANU [14], who uses the terminology Anderson-Brown-Cisinski premodel category.

Derivable categories are a natural generalisation of categories of fibrant objects in the sense of K. Brown [1, sec. 1]. More precisely: Given a derivable category, then its full subcategory of fibrant objects is a category of fibrant objects in this sense, and its full subcategory of cofibrant objects fulfills the dual properties.

In the proof of example 6.1, we have not used the existence of general finite limits and colimits [13, ch. I, $\S 1$, def. 1, ax. M0]. Moreover, to show that a Quillen model category carries the structure of a uni-fractionable category, we also did not use the lifting axioms [13, ch. I, $\S 1$, def. 1, ax. M1]. Thus we obtain the following more general example.

Example 6.3. We let $\mathcal{C}$ be a derivable category such that the following properties hold.

- Every identity in $\mathcal{C}$ is a cofibration and a fibration.

- Given an acyclic cofibration $i: X \rightarrow X^{\prime}$ and a morphism $f: X \rightarrow Y$ in $\mathcal{C}$, there exists a pushout rectangle in $\mathcal{C}$ as displayed below on the left, such that $i^{\prime}$ is an acyclic cofibration. Dually, given an acyclic fibration $p: Y^{\prime} \rightarrow Y$ and a morphism $f: X \rightarrow Y$ in $\mathcal{C}$, there exists a pullback rectangle in $\mathcal{C}$ as displayed below on the right, such that $p^{\prime}$ is an acyclic fibration.
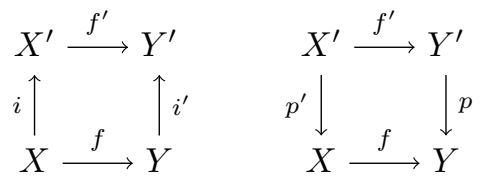

- For every weak equivalence $w: X \rightarrow Y$ in $\mathcal{C}$ there exists an acyclic cofibration 
$i: X \rightarrow Z$ and an acyclic fibration $p: Z \rightarrow Y$ with $w=i p$.

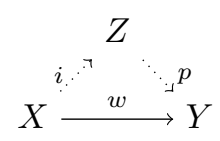

Then $\mathcal{C}$ carries the structure of a uni-fractionable category, where

$$
\begin{aligned}
\text { Den } \mathcal{C} & =\{w \in \operatorname{Mor} \mathcal{C} \mid w \text { is a weak equivalence }\}, \\
\text { SDen } \mathcal{C} & =\{i \in \operatorname{Mor} \mathcal{C} \mid i \text { is an acyclic cofibration }\}, \\
\text { TDen } \mathcal{C} & =\{p \in \operatorname{Mor} \mathcal{C} \mid p \text { is an acyclic fibration }\} .
\end{aligned}
$$

Proof. This is the same proof as for a Quillen model category, see part (a) of the proof of example 6.1 .

\section{References}

[1] Brown, Kenneth S. Abstract homotopy theory and generalized sheaf cohomology. Transactions of the American Mathematical Society 186 (1974), pp. 419-458.

[2] Cisinski, Denis-Charles. Catégories dérivables. Bulletin de la Société Mathématique de France 138(3) (2010), pp. 317-393.

[3] Deligne, Pierre. Cohomologie étale. Lecture Notes in Mathematics, vol. 569. Springer-Verlag, Berlin, 1977. Séminaire de Géométrie Algébrique du Bois-Marie (SGA4 $\frac{1}{2}$ ). With the collaboration of J.-F. Boutot, A. Grothendieck, L. Illusie and J.-L. Verdier.

[4] Dwyer, William G.; Hirschhorn, Philip S.; Kan, Daniel M.; Smith, JEFFREY H. Homotopy limit functors on model categories and homotopical categories. Mathematical Surveys and Monographs, vol. 113. American Mathematical Society, Providence (RI), 2004.

[5] Dwyer, William G.; Rădulescu-Banu, Andrei; Thomas, Sebastian. Faithfulness of a functor of Quillen. Algebraic \& Geometric Topology 10(1) (2010), pp. 525-530.

[6] Dwyer, William G.; Spaliński, Jan. Homotopy theories and model categories. Published as chapter 2 in [12, pp. 73-126], 1995.

[7] Gabriel, Peter; Zisman, Michel. Calculus of Fractions and Homotopy Theory. Ergebnisse der Mathematik und ihrer Grenzgebiete, Band 35. Springer-Verlag, New York, 1967.

[8] Gelfand, Sergei I.; Manin, Yuri I. Methods of homological algebra. Springer Monographs in Mathematics, second edition. Springer-Verlag, Berlin, 2003.

[9] Goerss, Paul G.; Jardine, John F. Simplicial Homotopy Theory. Progress in Mathematics, vol. 174. Birkhäuser Verlag, Basel, 1999.

[10] Gunnarsson, Thomas E.W. Abstract Homotopy Theory and Related Topics. Doctoral thesis, Chalmers University of Technology Göteborg, 1978. 
[11] Hovey, Mark. Model Categories. Mathematical Surveys and Monographs, vol. 63. American Mathematical Society, Providence (RI), 1999.

[12] James, IoAn M. (editor). Handbook of algebraic topology. North-Holland, Amsterdam, 1995.

[13] Quillen, Daniel G. Homotopical Algebra. Lecture Notes in Mathematics, vol. 43. Springer-Verlag, Berlin-New York, 1967.

[14] Rădulescu-Banu, Andrei. Cofibrations in Homotopy Theory. Preprint, 2006 (vers. 4, February 8, 2010). arXiv:math/0610009v4 [math.AT].

[15] Verdier, Jean-Louis. Catégoriés derivées. 1963. Published as appendix in [3, pp. 266-315], 1977.

Sebastian Thomas sebastian.thomas@math.rwth-aachen.de

Lehrstuhl D für Mathematik, RWTH Aachen University, Templergraben 64, 52062

Aachen, Germany 